\title{
OPTIMIZATION OF CLUSTERING AND COVERAGE OF TWO-TIER WIRELESS MESH NETWORKS
}

\author{
By \\ Sameer Qttlan \\ Bachelor of Applied Science \\ Electrical and Computer Engineering \\ King Abdulaziz University, Jeddah, Saudi Arabia, 2005 \\ A project \\ presented to Ryerson University \\ in partial fulfillment of the \\ requirements of the degree of \\ Master of Engineering \\ in the program of \\ Electrical and Computer Engineering
}

Toronto, Ontario, Canada, 2013

(C) Sameer Qttlan, 2013 


\section{AUTHOR'S DECLARAION FOR ELECTRONIC SUBMISSION OF A THESIS}

I hereby declare that I am the sole author of this thesis. This is a true copy of the thesis, including any required final revisions, as accepted by my examiners.

I authorize Ryerson University to lend this thesis to other institutions or individuals for the purpose of scholarly research.

I further authorize Ryerson University to reproduce this thesis by photocopying or by other means, in total or in part, at the request of other institutions or individuals for the purpose of scholarly research.

I understand that my thesis may be made electronically available to the public. 


\section{Abstract \\ Optimization of Clustering and Coverage of Two-Tier Wireless Mesh Networks \\ Sameer Qttlan, 2013 \\ Master of Engineering \\ Electrical and Computer Engineering \\ Ryerson University}

The tiered wireless mesh network is usually divided into upper and lower tiers. The upper tier is equipped with wireless optical transceivers; the lower tier connects the routers that connect the users. Users' connections can occur between each other or between nodes and outside the local area network in the lower tier. Whereas in the upper tier, the optical wireless communication is responsible for the higher level of data exchange. In this research, we apply a recent optimization technique for the design of wireless mesh access network in regards to the hop count and traffic demand requirements. This optimization is based on the plane sweeping and clustering algorithm (PSCA) to find the nodes and organize them in clusters based on location and distance. Then, we simulated the algorithm with Matlab software by generating a random network constellation and applied the algorithm to optimize the clustering of the network distribution. The simulations show the comparison between the number of clusters and their diameters. 


\section{Acknowledgements}

All thanks is due to Allah the most merciful and most compassionate. He guided me through my life as well as in my study and without him I would not be where I am now.

I would like to thank professor Xavier Fernando, my supervisor, for his precious time, efforts, guidance and patience through out my research.

I also would like to thank professor Kaamran Raahemifar for his support and encouragement towards my research. He has been very interested in my work and he always wanted my work to be better.

Many thanks to the government of Saudi Arabia and the Saudi Cultural Bureau in Ottawa for their sponsorship and continuous support for this study and research. Also, I would like to thank the General Authority of Civil Aviation of Saudi Arabia. They have helped me with their experience and for giving me this opportunity to pursue my studies.

I am grateful to my parents for their patience and love. I also would like to thank my sisters and my brother for being patient on me for being faraway form them for a long time. Many thanks to my friends and brothers back home in Saudi Arabia and here in Canada. They have been supporting me in completing this degree and without them I would be lost.

Finally, my wife and my daughter are the sole of this research and the motivation for my everyday life. They helped me all the way in my study and work to all of their best.

Toronto, ON

Sameer Qttlan

January, 2013 
To my parents. 


\section{Table of Contents}

Table of Contents $\quad$ vi

List of Tables viii

List of Figures $\quad$ ix

1 Introduction $\quad 1$

1.1 Motivation ...................... 5

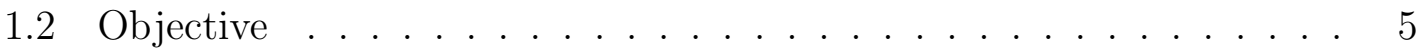

1.3 Assumptions . . . . . . . . . . . . . . . . . . . 5

1.4 Organization ......................... 6

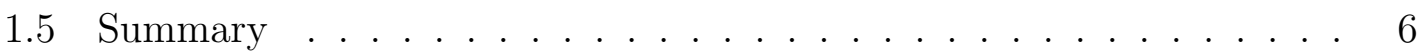

2 Background and Literature Survey 7

2.1 Wireless Mesh Networks . . . . . . . . . . . . . . . 7

2.2 Cellular Network . . . . . . . . . . . . . . . . . . . . . . . . . . . . . . .

2.3 Vehicular Wireless Network . . . . . . . . . . . . . . . . . . 10

2.4 Wireless Sensor Network . . . . . . . . . . . . . . . . 11

2.5 Clustering Optimization . . . . . . . . . . . . . . . . . . 12

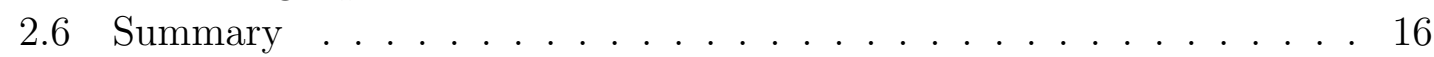

3 Optical Wireless Communication (OWC) 17

3.1 Introduction to Optical Communications . . . . . . . . . . . . . . 17

3.2 Free-Space OWC . . . . . . . . . . . . . . . . . . . 19

3.2.1 OWC Through Atmospheric Turbulence . . . . . . . . . . . . 20

3.2 .2 Urban OWC . . . . . . . . . . . . . . . . . 21

3.3 OWC Modulation with OFDM . . . . . . . . . . . . 23

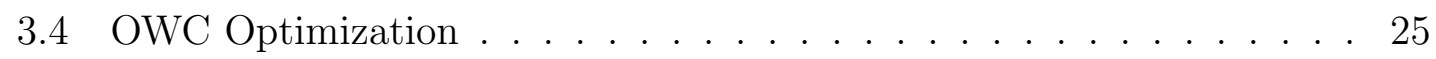

3.4.1 FSO Channel Model . . . . . . . . . . . . . . . 25

3.4.2 Optimizing the FSO Links . . . . . . . . . . . 26 
3.4.3 FSO Greedy Edge-Appending (GEA) . . . . . . . . . . 28

3.5 Summary . . . . . . . . . . . . . . . . . . . . 29

4 Plane Sweeping and Clustering Algorithm (PSCA) 31

4.1 Methodology . . . . . . . . . . . . . . . . . . 31

4.1.1 The Plane Sweeping and Clustering Algorithm (PSCA) . . . . 31

4.1 .2 Gravity Centre . . . . . . . . . . . . . . . . 35

4.2 Simulations . . . . . . . . . . . . . . . 35

4.2 .1 Simulation A . . . . . . . . . . . . . . 36

4.2 .2 Simulation B . . . . . . . . . . . . . . . . . 37

4.2 .3 Simulation C . . . . . . . . . . . . . . . . . . . . . 39

4.3 Results and Discussion . . . . . . . . . . . . . . . . 40

4.4 Summary . . . . . . . . . . . . . . . . . 43

5 Conclusion and Future Work 44

5.1 Conclusion . . . . . . . . . . . . . . . . . . 44

5.2 Future Work . . . . . . . . . . . . . . . . . . . 45

$\begin{array}{ll}\text { Bibliography } & 46\end{array}$ 


\section{List of Tables}

4.1 The plane sweeping and clustering algorithm (PSCA) . . . . . . . . 34

4.2 Cluster diameter and number of clusters. . . . . . . . . . . . . . . 41 


\section{List of Figures}

1.1 Global ICT developments, 2001-2011. . . . . . . . . . . . 2

1.2 Two-hop communication. . . . . . . . . . . . . . . 4

1.3 Tiered wireless network. . . . . . . . . . . . . . . . . . . . 4

2.1 Wireless mesh network $[1] \ldots \ldots$. . . . . . . . . . . . . 8

2.2 Evolution of cellular networks. . . . . . . . . . . . . . 10

2.3 Vehicle-to-infrastructure communication mode considered in the system model. . . . . . . . . . . . . . . . . . . . . . 11

2.4 Wireless sensor network. . . . . . . . . . . . . . . . 12

2.5 Son 175 nodes model. . . . . . . . . . . . . . . . . . . . . . . . 14

3.1 Standard wireless communication system. . . . . . . . . . . . 18

3.2 Weather effect $[2] \ldots \ldots \ldots \ldots$. . . . . . . . . . . . . . 19

3.3 Free space optical network [3]. . . . . . . . . . . . . . 20

3.4 Standard deviation of the log-amplitude fluctuation versus propagation distance. . . . . . . . . . . . . . . . . . . 22

3.5 Scattering medium in urban WOC. . . . . . . . . . . . . . 23

3.6 OFDM transmitter. . . . . . . . . . . . . . . . . 24

3.7 OFDM receiver. . . . . . . . . . . . . . . . . . . 24

3.8 Algebraic connectivity. . . . . . . . . . . . . . . 27

3.9 Algebraic connectivity vs. number of edges for small network. . . . . 29

3.10 Algebraic connectivity vs. number of edges for the large network. . . 30

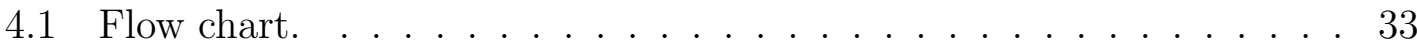


4.2 Simulation A: network distribution. . . . . . . . . . . . . . 36

4.3 Simulation A: clusters. . . . . . . . . . . . . . . . . . 37

4.4 Simulation B: network distribution. . . . . . . . . . . . . 38

4.5 Simulation B: clusters. . . . . . . . . . . . . . . . . . 38

4.6 Simulation C: network distribution. . . . . . . . . . . . . . . . . 39

4.7 Simulation C: clusters. . . . . . . . . . . . . . . . . . . . . 40

4.8 Number of clusters vs cluster diameter for 20 nodes network. . . . . . 41

4.9 Number of clusters for 50 nodes. . . . . . . . . . . . . . . . . . 42

4.10 Number of clusters for 175 nodes. . . . . . . . . . . . . . . . . . . 43 


\title{
List of Symbols
}

\author{
Symbol Description \\ $n_{c} \quad$ Number of clusters. \\ $y_{i} \quad$ Binary variable for the router count. \\ $x_{i j} \quad$ Indicator if the router $i$ is associated with router $j$. \\ $h_{i j} \quad$ Hop count between router $i$ and $j$. \\ $f_{M_{i}} \quad$ Traffic of the cluster $M_{i}$. \\ $Y_{i} \quad$ Mean number of $M$ nodes per cluster $N$ for $j$ number of clusters. \\ $\rho \quad$ Coefficient relationship among the nodes inside the cluster. \\ $f \quad$ Sampling fraction. \\ C Cost. \\ V Variance. \\ $P_{R} \quad$ Power received after scattering. \\ $\varphi \quad$ Field of view angle. \\ $\delta \mathrm{s} \quad$ Pointing error. \\ $\tau \quad$ Optical density. \\ Z Transmission range. \\ $\lambda$ Radiation wavelength. \\ A Aperture size. \\ $Q \quad$ Received scattered light. \\ $F_{I} \quad$ Received light intensity.
}


$I_{0} \quad$ Average received intensity.

$\sigma_{x}^{2} \quad$ Variance of the log-amplitude uctuation.

$C_{n}^{2}(\eta) \quad$ Parameter of the refraction structure.

$\eta \quad$ Constant altitude.

$z \quad$ Transmission distance for atmospheric channels near the ground.

$\Gamma_{i j} \quad$ Reliability of the FSO link.

$D \quad$ Graph diameter.

$\delta(G) \quad$ Minimum degree of the graph.

$K_{i} \quad$ Degree of each cluster head vertex $v_{i} \in V$.

$E_{\text {opt }} \quad$ Potential edges (cluster heads). 


\section{List of Abbreviations}

$\begin{array}{ll}\text { Abbreviation } & \text { Description } \\ 2 \mathrm{G} & \text { Cellular second generation. } \\ 3 \mathrm{G} & \text { Cellular third generation. } \\ \text { ASK } & \text { Amplitude shift keying. } \\ \text { BS } & \text { Base station. } \\ \text { CDMA } & \text { Code division multiple access. } \\ \text { DD } & \text { Direct detection. } \\ \text { DVB } & \text { Digital video broadcasting. } \\ \text { FSO } & \text { Free space optical. } \\ \text { GEA } & \text { Greedy edge-appending. } \\ \text { GPS } & \text { Global positioning system. } \\ \text { HDTV } & \text { High-definition TV. } \\ \text { ICI } & \text { Intercarrier interference. } \\ \text { IM } & \text { Intensity modulation. } \\ \text { IP } & \text { Internet protocol. } \\ \text { ISI } & \text { Intersymbol interference. } \\ \text { ISP } & \text { Internet service provider. } \\ \text { IEEE } & \text { Institute of electrical and electronics engineers. } \\ \text { LAN } & \text { Local area network. } \\ \text { LED } & \text { Light emitting diode. } \\ \text { LOS } & \text { Line of sight. }\end{array}$


LTE Long term evolution.

MAC Media access control.

MT Mobile terminal.

OFDM Orthogonal frequency division multiplexing.

OOK On-off keying.

OPA Optical phased arrays.

OWC Optical wireless communication.

PSCA Plane sweeping and clustering algorithm.

PSK Phase shift keying.

QAM Quadrature amplitude modulation.

RSU Road-side unit.

UOWC Urban Optical wireless communication.

V2V Vehicle to vehicle.

VANET Vehicular ad hoc communication network.

WAVE Wireless access in vehicular environment.

WLAN Wireless local area networks.

WMAN Wireless metropolitan area networks.

WMN Wireless mesh network.

WPAN Wireless personal area networks.

WSN Wireless sensor network.

WWAN Wireless wide area networks. 


\section{Chapter 1}

\section{Introduction}

Wireless communication is the most rapidly growing technology in the physical sciences. There were 11 million wireless mobile subscribers in 1990 and 6 billion by the end of 2011 [4]. Figure 1.1 shows the development in the use of wireless networks. It distinguishes the types of services in the number of users over time. Moreover,Internet use has become the most in-demand service in the world. This aggressive demand for the wireless services is increasing with the emergence of new applications [5]. It is used in health applications, sensors and in smart buildings and environments $[6]$.

Obtaining an organized hierarchical network is a known important problem in wired networks. However, in wireless networks, clustering the nodes into groups is a very similar problem. Clustering the network well control the spatial reuse of the shared channel, minimize the bandwidth of the exchanged data and build and maintain the architecture of the network. The fully distributed linked cluster architecture was developed for routing and demonstrating the network connectivity $[7]$.

The power consumption and bandwidth utilization are the two major challenges for the wireless networks. To reduce the power consumption we need an efficient 


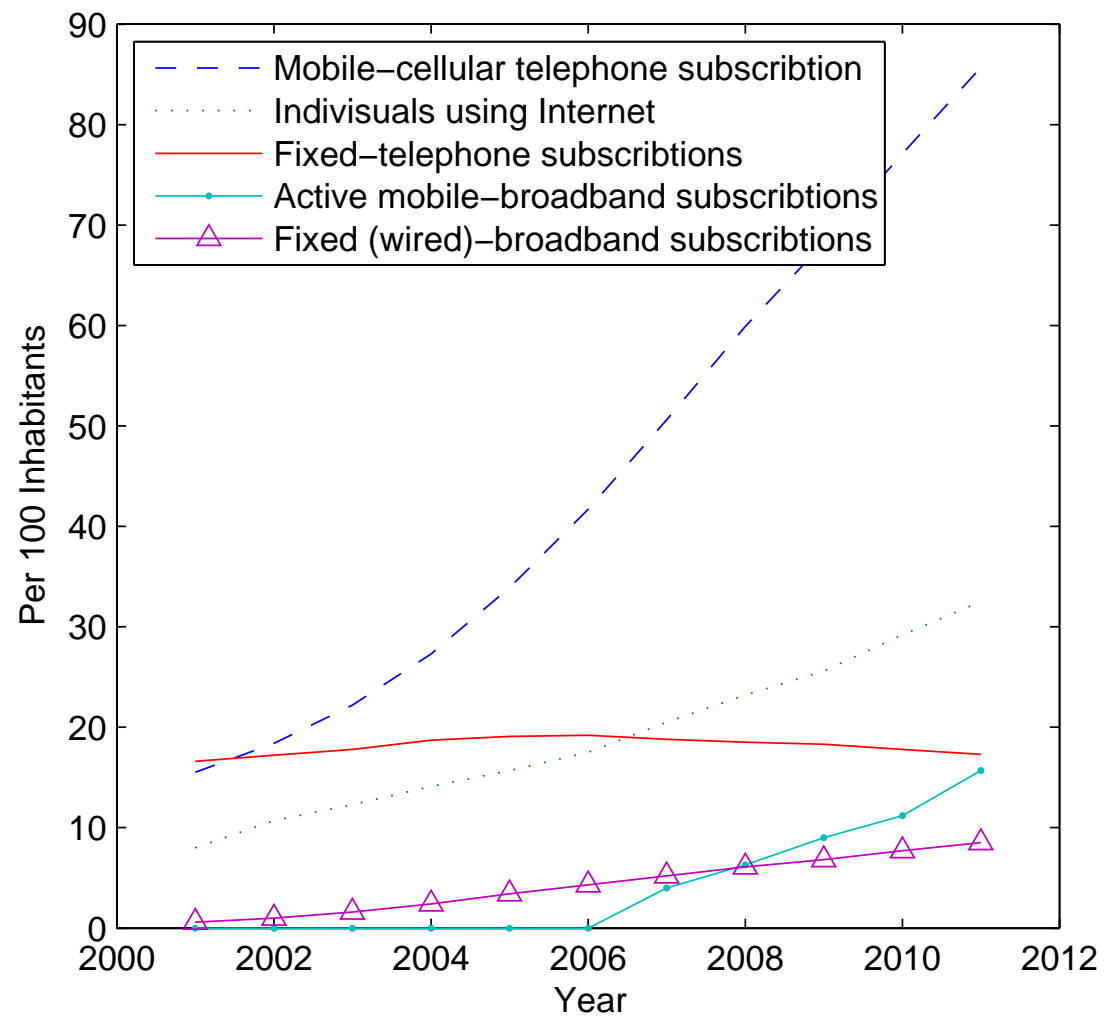

Figure 1.1: Global ICT developments, 2001-2011.

techniques for the data transmission or by reorganizing the network structure. A similar concept has to be applied for the bandwidth utilization as well [8].

In this project we will focus on the clustering optimization. Each cluster wight the nodes in the cluster and chooses the best one to be the head node. The nodes are distributed randomly and they should be cluster into several groups to ensure the power will be saved and the bandwidth will be utilized. Moreover, the multi-hop communication is a promising approach to achieve more reliability.

There are several types of wireless communication networks, as defined by the standardizing committees of the Institute of Electrical and Electronics Engineers (IEEE) [9]: 
- Wireless Local Area Networks (WLAN)

WLAN is used when connecting devices in a small and circumscribed area like a building. It includes a distribution point [10].

- Wireless Personal Area Networks (WPAN)

WPAN connects a limited number of users without a connection to the outside world. It is a secure and manageable network [11].

- Wireless Metropolitan Area Networks (WMAN)

This is also referred to as WiMax. It covers a big network over a large area and connects different WLANs [12].

- Wireless Wide Area Networks (WWAN)

WWAN can cover very large areas such as cities.

The Multi-hop connection applies when we use one node to act like a bridge in order to connect two nodes that are separated by a larger distance. For example if nodes $\mathrm{A}$ and $\mathrm{B}$ are separated by 10 meters and node $\mathrm{C}$ is separated by 20 meters from $\mathrm{A}$ but 5 meters from $\mathrm{B}$, and if the connection limit is 10 meters, then the A and $\mathrm{C}$ cannot communicate. Therefore, the multi-hop connection from $\mathrm{A}$ to $\mathrm{B}$ to $\mathrm{C}$ will allow this communication to go through [13]. See figure 1.2.

The multi-hop technique has been used for a long time to improve the reliability and availability of communication. It has been used to improve the performance of cellular networks [14]. This technique has increased network coverage in an area and reduced power consumption.

In our model we will introduce the use of two-hop communication in the clustering process. The algorithm will consider the nodes within the specified conditions and the nodes within two-hop range. We will apply the tiered wireless network in 


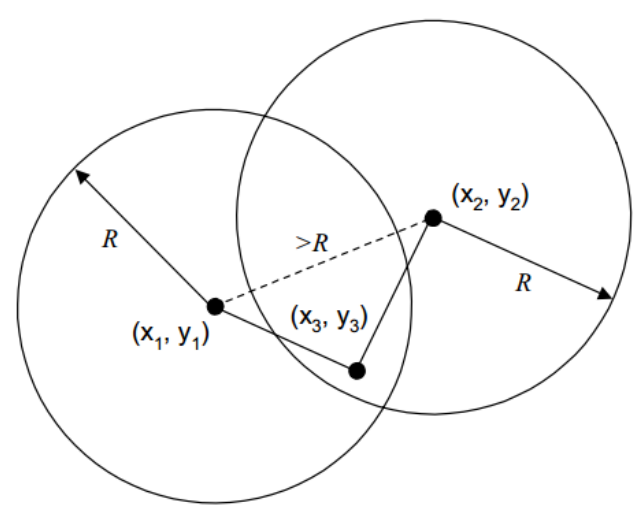

Figure 1.2: Two-hop communication.

our example and to the reference problem [15]. Figure 1.3 shows the proposed network scheme by Son [15]. The wireless optical links represent the head of the cluster for the lower tier and they tried to minimize the number of clusters to reduce the number of the wireless optical links.

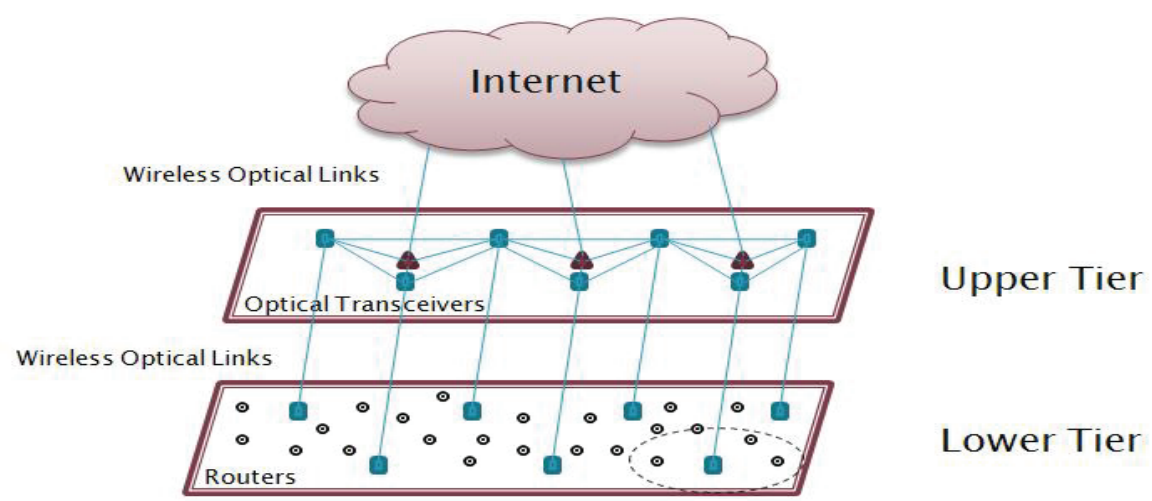

Figure 1.3: Tiered wireless network. 


\subsection{Motivation}

The increasing demand for wireless services and applications is growing rapidly. Therefore, in this research we hope to increase the wireless network capacity in physical positioning. The goal of this research is to optimize the number of clusters for a given network distribution based on the number of nodes and the coverage distance for each cluster head. We will then choose the centre of gravity theory to choose the cluster head of each group of nodes. Using fewer head clusters will result in fewer base stations to ensure coverage for all the nodes. Therefore, the cost will be minimized to the optimum.

\subsection{Objective}

The main objective of this project is to reduce the power consumption and the bandwidth utilization. The clustering will be an efficient technique for reorganizing the network structure. Therefore, we will try to develop an optimization technique for clustering the wireless network.

\subsection{Assumptions}

Our assumptions are as follows:

- Increasing the coverage distance of the base station will result in a larger cluster and larger coverage area.

- Using larger clusters will minimize the number of cluster heads and therefore fewer base stations will be needed to cover the whole area. 
- Using fewer clusters will result in greater power consumption with higher power levels in the base stations.

These assumptions were based on the research of [16].

\subsection{Organization}

The report is organized in the following chapters:

- Chapter 2: Literature Survey. In this chapter we will define the terms we are using in this project. Moreover, this chapter will discuss previous work on the optimization of wireless networks in different areas.

- Chapter 3: Optimization and Results. This chapter will present the algorithm used in this project, modulations and experiments of the code, the results and the outcomes.

- Chapter 4: Discussion and Future Work. We will discuss the techniques that can be used in optimizing a wireless network. Then, a summary of the results and a comparison of the various techniques will be presented.

\subsection{Summary}

In this chapter, we introduced the main elements of the research. We explained the importance of the wireless networks and their applications. Then, we defined our objective and the purpose of the research. Also, the assumptions have been cleared. In the next chapter, we will present the background of the topic and the literature survey. 


\section{Chapter 2}

\section{Background and Literature Survey}

\subsection{Wireless Mesh Networks}

Wireless mesh networks (WMN) have been increasing in popularity in the last two decades. They have emerged as a promising technology for providing reliable wireless access for mobile users, and an improvement for the local area networks into WLAN. Therefore, WMN have been the focus of a considerable amount of research and development [17][15]. One of the major shortcomings of WMN is scalability. The scalability problem is defined as the optimization problem between throughput and number of nodes. In [18], they showed that the throughput is proportional to the number of nodes and therefore the throughput decreases by this relation:

$$
O(1 / n)
$$

, where $\mathrm{n}$ is the number of nodes.

Moreover, [19] by Shi et. al. showed the difference between the single and multihop flow and need for bandwidth. A network uses MAC protocols to communicate and exchange data over wired and wireless networks. The results showed that the 


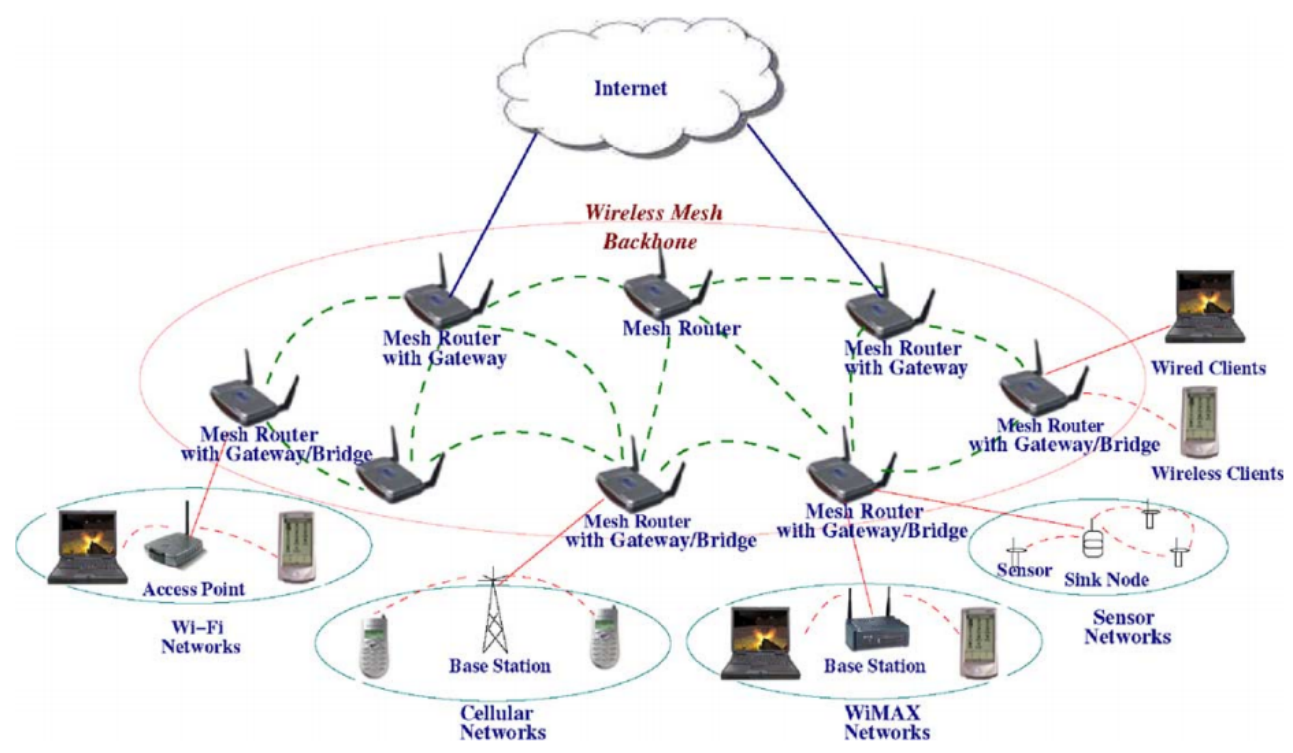

Figure 2.1: Wireless mesh network [1].

network behavior is affected by the policy of the network distribution. This occurs because of the inefficiency and the bi-stability of the MAC protocols. Therefore, a hierarchical architecture is needed for the wireless network to provide a powerful and more throughput networks [20].

In [21], it is shown that the asymptotic throughput increases by a linear relationship with the number of base stations that are connected through wired links that have high bandwidths. This happens if the number of base stations grows faster than $\sqrt{n}$. See figure 2.1 .

\subsection{Cellular Network}

The increasing popularity of wireless communication networks has demonstrated that customers are expecting the same level of service and availability (see figure 2.2). They expect the performance of wireless communication networks to be as the 
traditional wire-line networks, which has generated the need for more advanced research to achieve the same quality of service. Advancement of wireless technologies (2G, 3G, WLAN, WMAN, etc.) in combination with the development of Mobile Terminals (MTs) with multiple network interfaces and the evolution of IP-based applications (non-real-time or real-time), has given users access to mobile services anywhere at any time from any network. An additional revolutionary step, driven by this universal wireless access, is the fourth generation $(4 \mathrm{G})$ and Long Term Evolution (LTE) of cellular networks [22] [23]. This next-generation of cellular networks exhibits a heterogeneous environment with different access networks technologies that vary in bandwidth, latency and cost [24]. In this environment, mobility management is the mandatory issue that facilitates the roaming of users from one network to another. Handoff management, one of the components of mobility management, controls the change of the MTs point of attachment during active communication. Handoff management issues include mobility scenarios, metrics, decision algorithms and procedures. Mobility scenarios can be categorized as horizontal (among different clusters of the same network) and vertical (among different types of networks) $[25]$.

The major challenges in vertical handover are seamlessness and automation in network switching. These challenges are better known as "Always Best Connected" in a scenario of multiple access technologies, according to network and user policies [26]. These policies are expressed by rules based on parameters such as network conditions or user preferences [27]. For that, a handoff management technique must select the appropriate time to initiate the handoff and the most suitable access network for a service among those available, and must maintain call or service continuity. 


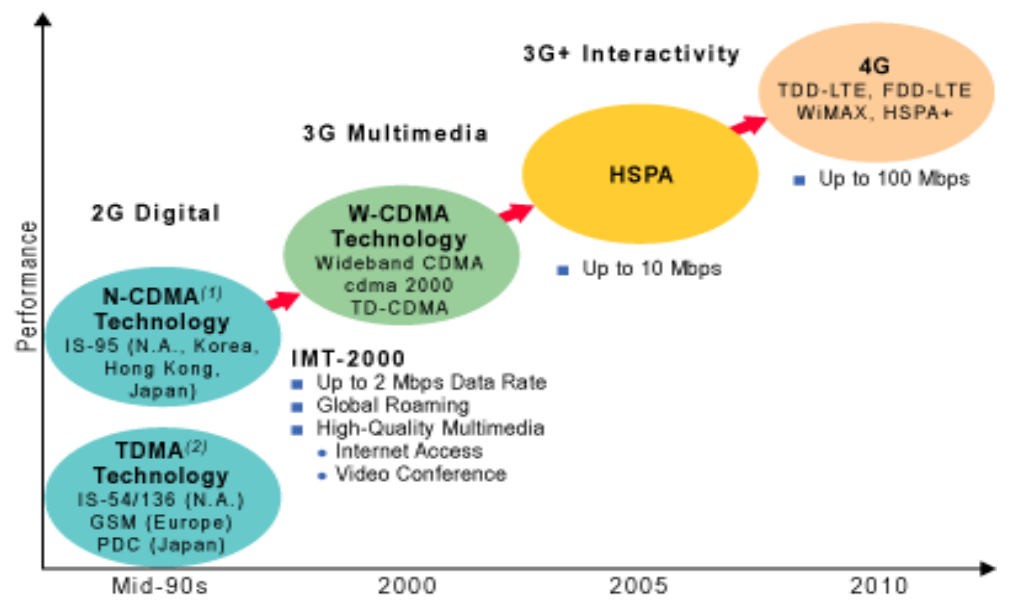

Figure 2.2: Evolution of cellular networks.

\subsection{Vehicular Wireless Network}

Vehicular wireless networks have elicited a vast amount of interest from industry in the last decade [28]. The fact that the European vehicle manufacturers has been initiated a nonprofit vehicle to vehicle $(\mathrm{V} 2 \mathrm{~V})$ communication systems to support safety system and other applications [29]. In July 2010, IEEE research publications publications developed this standard and it was then called, IEEE 802.11p Medium Access Control Sub-layer and Physical Layer were certificated to support Wireless Access in Vehicular Environments (WAVE) [30][10]. These developments have led to increasing focus on the safety communications that related to exchange of packets to alert vehicles about an imminent collision or dangerous situation [31]. In Vehicular Ad Hoc Communication Networks (VANETs), vehicles communicate with Road-Side Units (RSUs), referred to as Vehicle-to-Infrastructure (V2I) communications (figure 2.3). Moreover, vehicles can communicate with each other using (V2V) communications. The applications of VANETs range from strict safety to information and entertainment services [32]. 


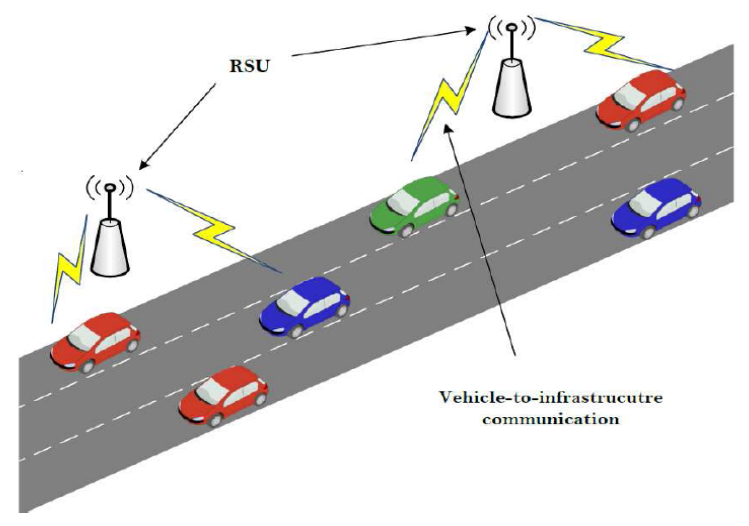

Figure 2.3: Vehicle-to-infrastructure communication mode considered in the system model.

\subsection{Wireless Sensor Network}

The nodes in a wireless sensor network are connected wirelessly to measure signals. These signals can be out of heat measure, motion or light. The sensor are usually capable of collecting, storing and/or communicating. This type of network is very popular because of the simplicity of the sensors and low cost. Wireless sensors are commonly used, among others, in fire detection, gas alerts, temperature control [33] [34]. 


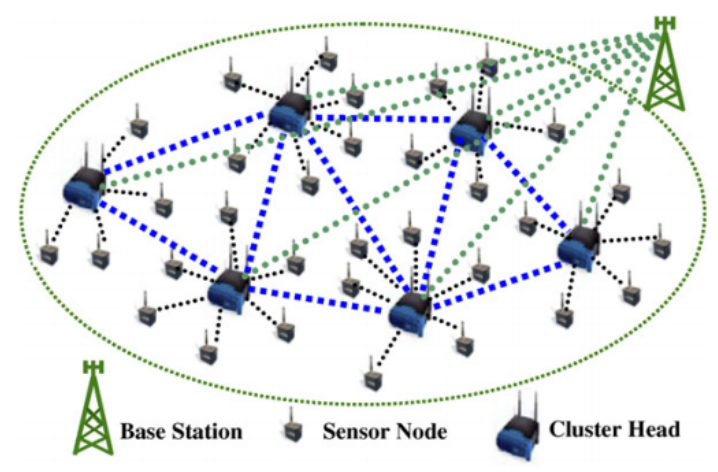

Figure 2.4: Wireless sensor network.

A wireless sensor network is another type of wireless networks that can be used as an application for our algorithm. Clustering these types of networks will reduce the number of sensors required to cover the area and will also the need to connect additional base stations to the main control units [35]. In [36], they introduced a mechanism to cluster the wireless sensors into different groups in order to reduce the number of nodes connected to a single hub. In this case they can track and monitor the position of a signal from a single sensor or node. They used a hierarchical algorithm and a new protocol for the MAC layer to protect and secure the data transmitted into the network. Figure 2.4 shows a simple wireless sensor network.

\subsection{Clustering Optimization}

Son and Mao paper [15] uses the Plane and Sweeping algorithm to minimize the number of clusters. This technique starts with a node and makes it as the primary one. Then, it sweeps the nodes and select the first one and assign it with the first router. Moves to the next one, if it is not assigned to a router, it will assign it to the same router. Then, the algorithm continues as long as the conditions not violated. 
If so, the algorithm will start another cluster.

Objective function:

Minimize:

$$
n_{c}=\sum_{i=1}^{n} y_{i}
$$

It is subject to these conditions:

$$
\begin{gathered}
\sum_{j=1}^{n} x_{i j}=1 \quad(\text { for all } i) \\
\sum_{j=1}^{n} x_{i j} \cdot h_{i j} \leq h_{\text {max }} \quad(\text { for all } i) \\
f_{M_{i}} \leq f_{\max } \quad\left(\text { for all } M_{i}\right) \\
x_{i j} \in\{0,1\}, y_{i} \in\{0,1\} \quad(\text { for all } i, j)
\end{gathered}
$$

, where:

$n_{c}:$ is the number of clusters.

$y_{i}$ : is binary variable for the router count.

$x_{i j}$ : is an indicator if the router $i$ is associated with router $j$.

$h_{i j}$ : is the hop count between router $i$ and $j$.

$f_{M_{i}}$ : is the traffic of the cluster $M_{i}$.

The method depends on the plane sweeping and clustering algorithm to optimize the cluster size. The authors used a model with these specifications:

1. Number of nodes $=175$

2. Network size $=1000 \times 1000$ 
3. Maximum cluster diameter ranging [100 - 1000]

The results can be shown from their paper in figure 2.5. This will be compared with our results in the next chapter.

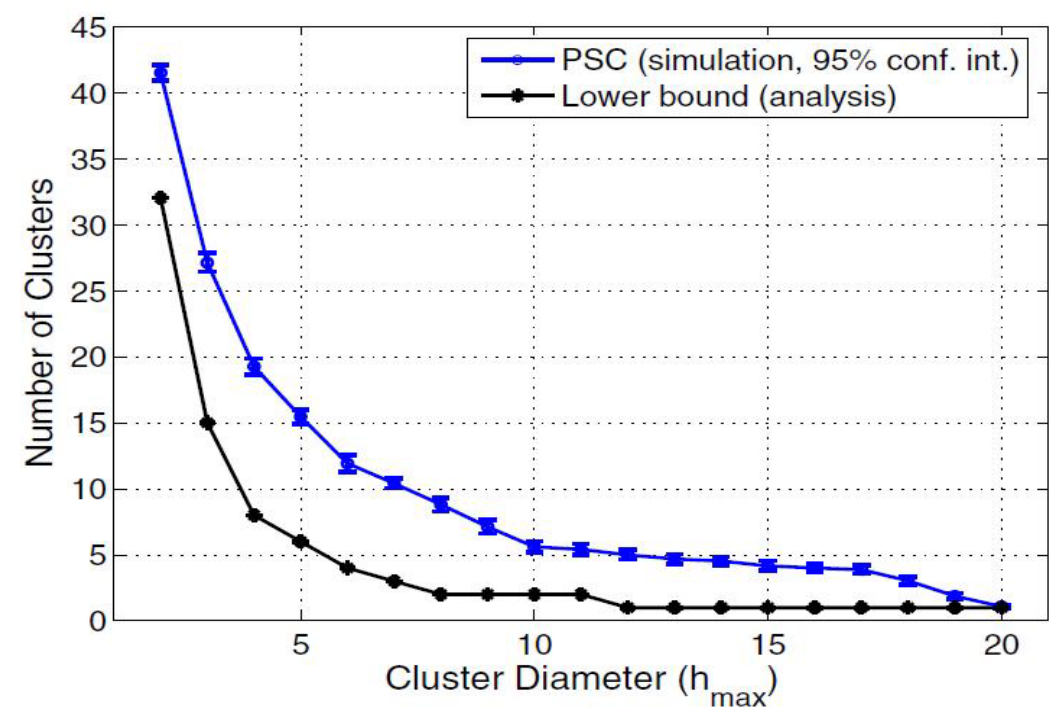

Figure 2.5: Son 175 nodes model.

In this paper [37], Dutta, Gupta and Das introduced a randomly distributed technique to optimize the distribution of the nodes into clusters. Their objective was to find the optimum size and cost of the cluster and to organize the nodes within the cluster. When clusters were identical, the mean number of $M$ nodes per cluster $N$ for $j$ number of clusters is given by the following formula:

$$
Y_{i}=\sum_{j=1}^{M} \frac{Y_{i j}}{M}
$$

The next formula shows the relationship among the nodes inside the cluster by defining the $\rho$ coefficient: 


$$
\rho=\frac{E\left(y_{i j}-\bar{Y}\right)\left(y_{i k}-\bar{Y}\right)}{E\left(y_{i j}-\bar{Y}\right)^{2}}
$$

They also defined $n$ as a group of identical clusters from the whole $n$. Therefore, the sampling fraction can be expressed as $f=n / N$.

The cost function can be simplified by the following formula:

$$
C=c_{1} n M+c_{2} n^{\frac{1}{2}}
$$

Where $c_{1}$ and $c_{2}$ are the cost of the moving nodes inside the cluster and between the neighbor clusters respectively. The total number of nodes in a cluster $n$ out of $N$ clusters would be:

$$
\bar{Y}_{c}=N M \bar{Y}_{n}
$$

and the variance would be as follows:

$$
\begin{gathered}
\bar{Y}_{c}=(N M)^{2} V\left(\bar{Y}_{n}\right) \\
V\left(\bar{Y}_{c}\right) \cong M N^{2} \frac{1-f}{n} S^{2}[1+(M-1) \rho]
\end{gathered}
$$

In this paper, the authors have shown from the above equations that the variance of the nodes in all clusters is higher than the the one of a a sample of clusters. Moreover, they proved that decreasing the mean square between clusters would increase the efficiency. The cost can be optimized by minimizing the cost function above. Therefore, decreasing the variation between clusters would increase the efficiency of the network. 


\subsection{Summary}

In this chapter, we increase our knowledge about the clustering of the wireless networks. We also discussed some of the applications where this algorithm can be implemented, such as WMN, cellular network, vehicular network and wireless sensor networks. Moreover, we reviewed some of the techniques that are being used to optimize the clustering in the wireless networks. 


\section{Chapter 3}

\section{Optical Wireless Communication (OWC)}

\subsection{Introduction to Optical Communications}

Optical communication is the most prominent communication system among all others because it is the fastest transmission system known to the humanity. There are several types of optical communication systems. Optical fiber is a widely known system that uses LED or Lasers to modulate the digital signal into the fiber. Because this type of optical communication depends on coherent light sources, it has the highest information capacity among the other systems. Moreover, this type provides very reliable transmission over very long distances with low losses to a few $d B s / \mathrm{km}$. It is directly affected by the impurities in the fiber material [38].

The standard wireless communication system for digital communication still applies to the optical communication link. The system receives the analog signal, and then it is converted into digital using the $A / D$ converter. The signal is then encoded (or quantized) into a scheme to make it ready for transmission. Then the signal is modulated with a modulation scheme (PSK, ASK, CDMA, OFDM), and it goes back to analog for transmission by antenna or light source. In real life, the 
transmitted signal travels through a wireless channel with different types of noises and losses. The channel is affected by the normal noise of the space. Moreover, shadowing and fading are the two main concerns in wireless communication. In the receiver, the signal is received by an antenna of a photodetector in the optical case. Then it is demodulated and decoded. It then must be converted back into analog through a $D / A$ converter. Finally, the signal is received with errors and noises. Filters and gains can be used to overcome some of these issues. Figure 3.1 shows this digital communication link.

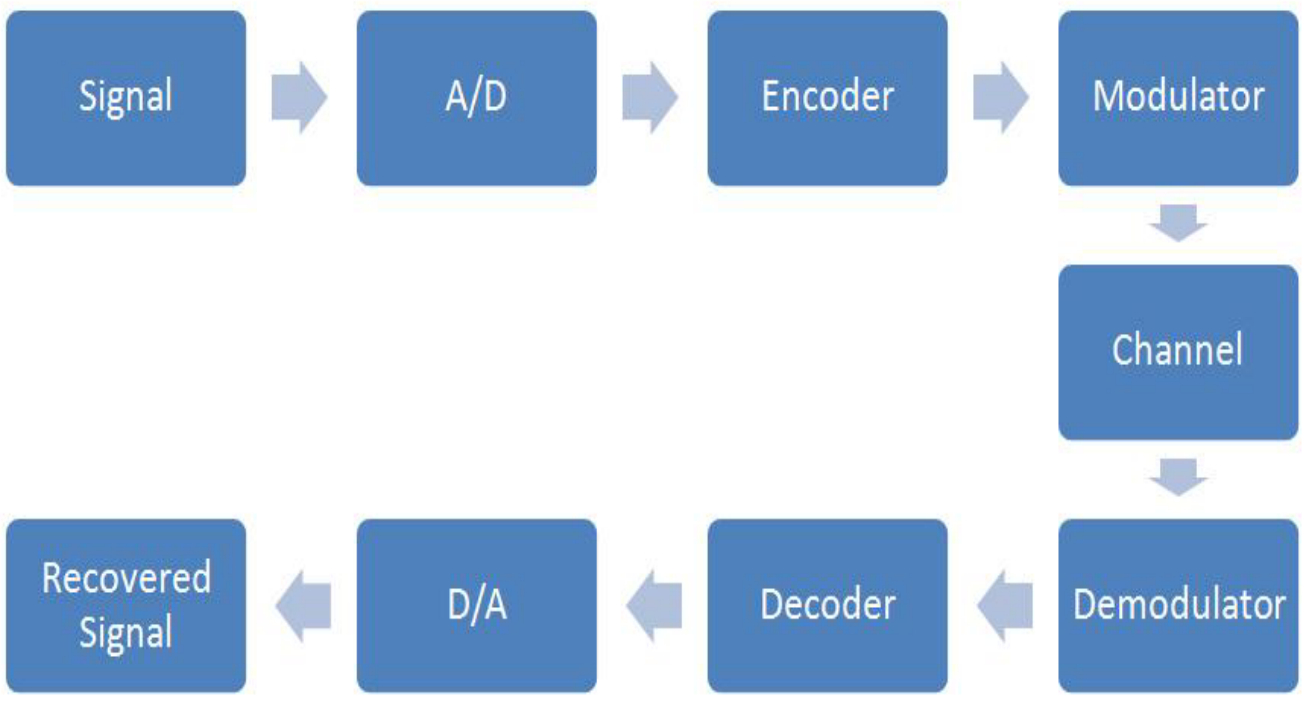

Figure 3.1: Standard wireless communication system.

Optical wireless communication is another type of optical communication. It is also referred to as free-space laser communication. This type can be used for indoor applications as well at $12.5 \mathrm{GHz}$. [39]. With this type, the transmitter and the receiver (photodetector) are separated by distance and communicate through air or free space. There are concerns about the reliability of the service because of the interruption of the line of sight in the horizontal links and the weather conditions 
in the horizontal and vertical links. The changing of wavelength in the light source does not eliminate the effect of weather on the wireless communication link [2]. See fig.3.2.

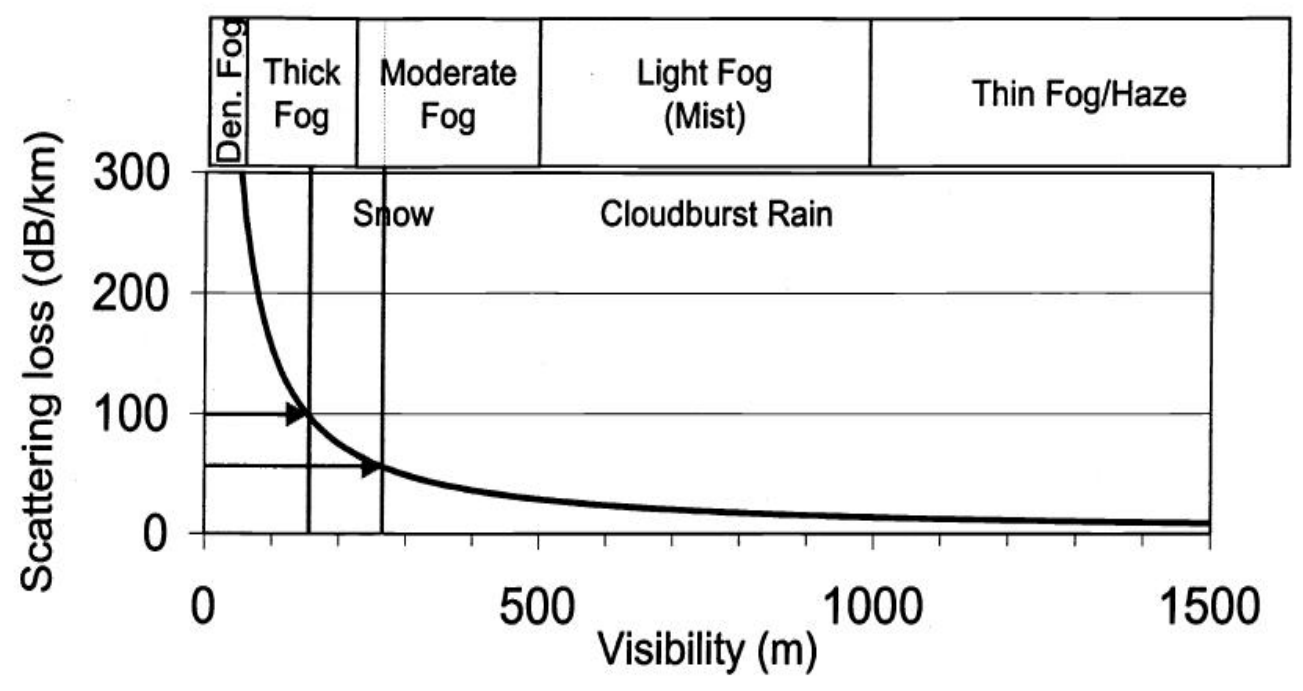

Figure 3.2: Weather effect [2].

\section{$3.2 \quad$ Free-Space OWC}

Free space optical communication presents a promising future for wireless networks. Wireless optical communication has the potential to solve the problem of high capacity and cost savings as compared to typical wireless links. We have to take into account the design of inexpensive and simpler receivers to meet the operation constraints. Operating high-level power sources safely in indoor environments is a major challenge that can affect the performance of wireless optical systems. See Figure 3.3. 


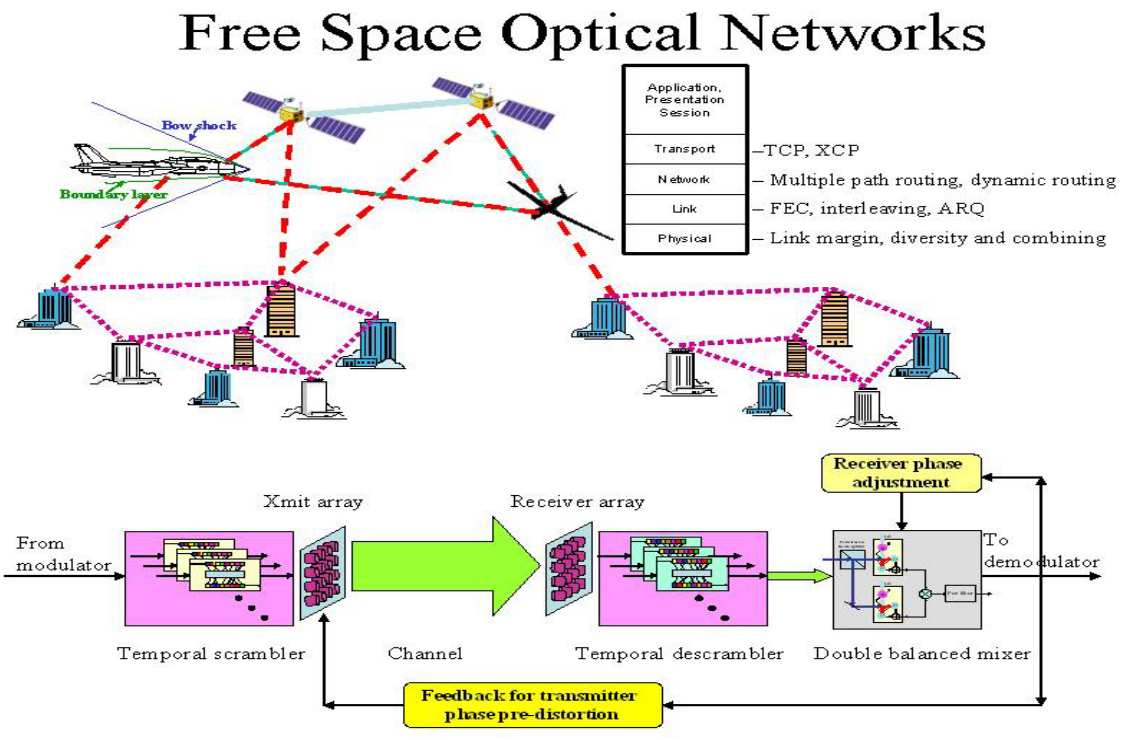

Figure 3.3: Free space optical network [3].

In [40], they have revealed five major challenges in free-space wireless communication:

1. Applying free space optics as a wireless network solution.

2. Using wireless optical as a solution to the rst mile problem.

3. Delivering the promised communication capacity to end users in indoor environments.

4. Designing simpler receivers that meet the constraints.

5. Using high-level power sources while ensuring human safety.

\subsubsection{OWC Through Atmospheric Turbulence}

Free space optical communication systems use intensity modulation with direct detection (IM/DD). However, in distances of more than $1 \mathrm{~km}$, the performance of the 
link is degraded because of atmospheric turbulence. The turbulence is affected and measured by two factors: the correlation length of intensity fluctuations $d_{0}$, and the correlation time of intensity fluctuations $\tau_{0}$. We will also define the receiver aperture $D_{0}$ that can be larger than $d_{0}$, and therefore the fading introduced by the turbulence can be decreased by averaging the aperture [41]. Assuming that $D_{0}<d_{0}$ and the receiver observation interval $T_{0}<\tau_{0}$, two techniques can be considered; temporal-domain and spatial-domain.

In [42], Zhu et. al. studied turbulence-induced fading based on statistical distributions. The reception of the spatial diversity can also help to mitigate turbulenceinduced fading. When the fading correlation length is not lower than the difference between receivers, correlation reduces the diversity gain, but their technique can overcome some of this loss. Figure 3.4 shows the standard deviation of the logamplitude fluctuation and the propagation distance where $C_{n}$ is the wavenumber spectrum structure parameter.

\subsubsection{Urban OWC}

The urban OWC (UOWC) is the local version of the OWC. It is installed on a range of hundreds of meters and requires a line of sight for the communication link to work. The line of sight is also essential for outdoor UOWC. This can be maintained through the help of tracking and pointing systems. Tracking is performed in two stages:

1. Coarse tracking: Accomplished by using GPS or determined locations.

2. Fine pointing: Accomplished through the use of electro-optic mechanisms that have a beam-steering device such as galvo-mirror or optical phased arrays (OPAs). 


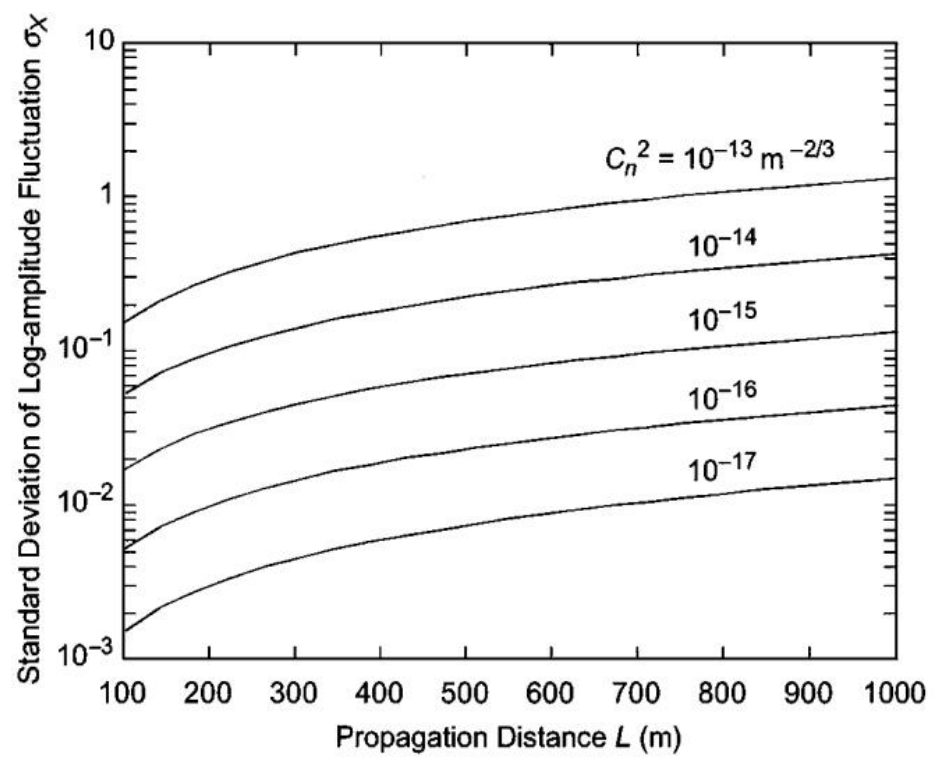

Figure 3.4: Standard deviation of the log-amplitude fluctuation versus propagation distance.

The link between transmitter and receiver can be extended to a network. This allows for more flexibility and extends the transmission range [43]. The channel can be scattered between the transmitter and the receiver, and the power received after scattering can be estimated by the following formula:

$$
P_{R}(\varphi, \delta, \tau, \lambda, A)=\int_{\delta-\varphi / 2}^{\delta+\varphi / 2} Q(\varphi, \tau, \lambda, A) d \varphi
$$

where $\varphi$ is the field of view angle, $\delta$ is the pointing error, $\tau$ is the optical density (dened as $Z / D$, where $Z$ is the transmission range), $\lambda$ is the radiation wavelength, $A$ is the aperture size, and $Q$ represents the received scattered light. Figure 3.5 shows the scattering in the presence of the line of sight in the UOWC [44]. 


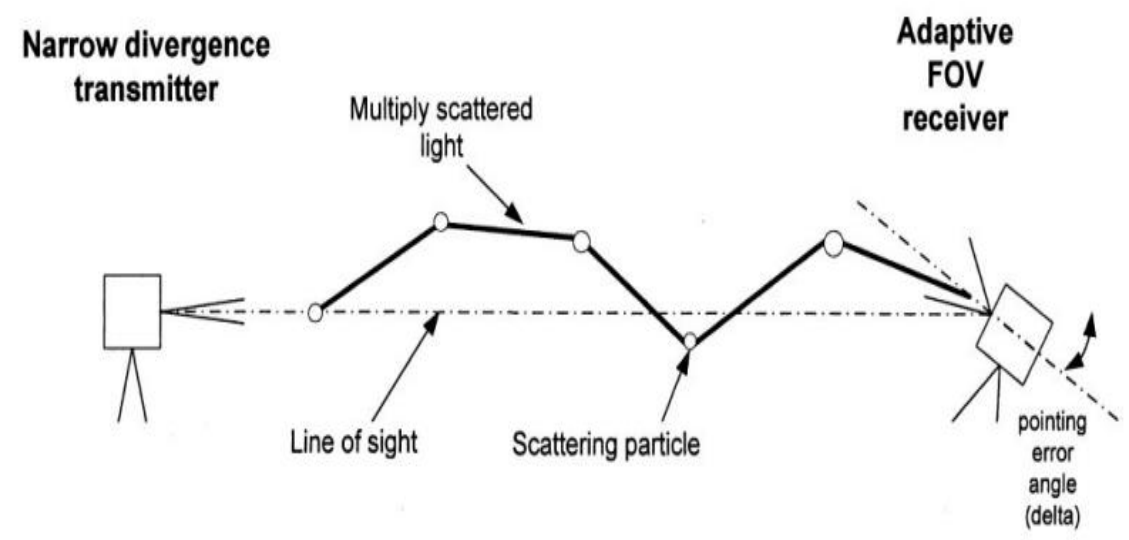

Figure 3.5: Scattering medium in urban WOC.

\subsection{OWC Modulation with OFDM}

Orthogonal frequency division multiplexing is a multicarrier modulation technique that has recently found wide adoption in a variety of high-data-rate communication systems. Some of the examples of these systems are DSL, wireless LAN, DVB, and WiMAX. OFDM technology has been used in optical fiber and optical access networks for long time. The performance of the OFDM over fiber has a high spectrum efficiency. Today, the demand is rising for bandwidth. The demand now is more than $10 \mathrm{~Gb} / \mathrm{s}$ for very high speed Internet (usually service providers ISPs) and highdefinition TVs (HDTV) in homes. Therefore, more advanced techniques are needed to overcome these demands.

The old OFDM technology used quadrature amplitude modulation (QAM) or phase shift keying (PSK) with different orders of M. These two techniques were successfully implemented and worked for the lower speeds. However, increasing the speed would result in two major drawbacks. First, the power consumption would 
be very high. Second, the intercarrier interference (ICI) would also increase. Due to these two factors, the receiver would be more complicated and expensive [45][46]. Figures 3.6 and 3.7 shows a standard OFDM transmitter and receiver.

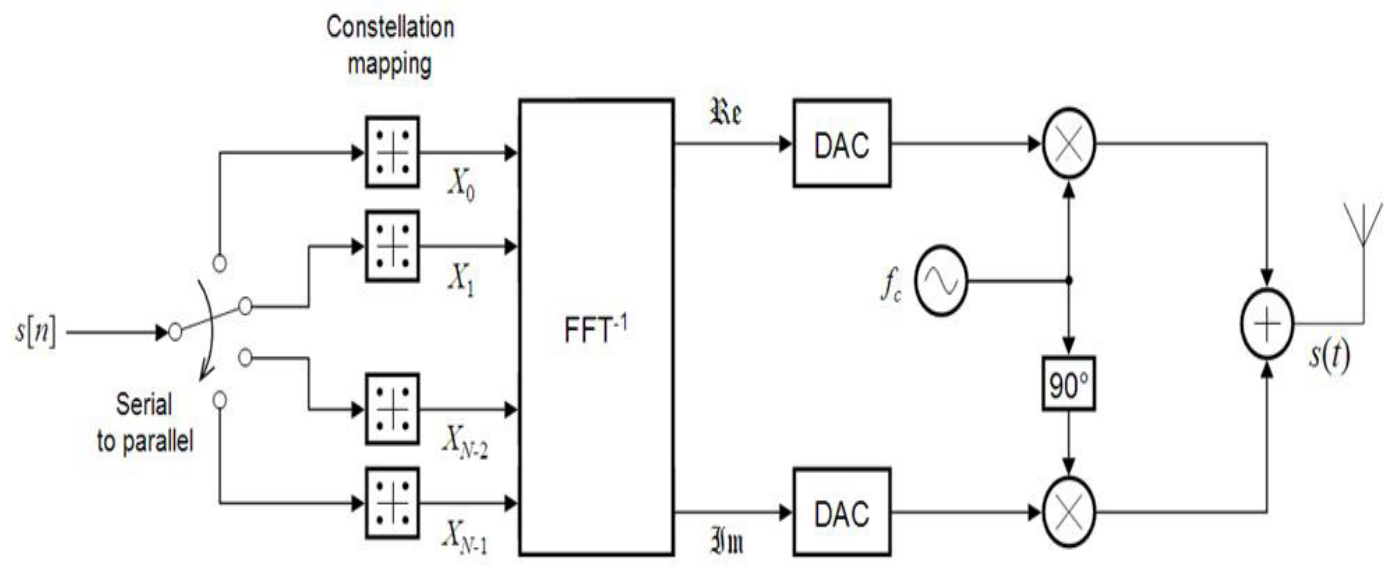

Figure 3.6: OFDM transmitter.

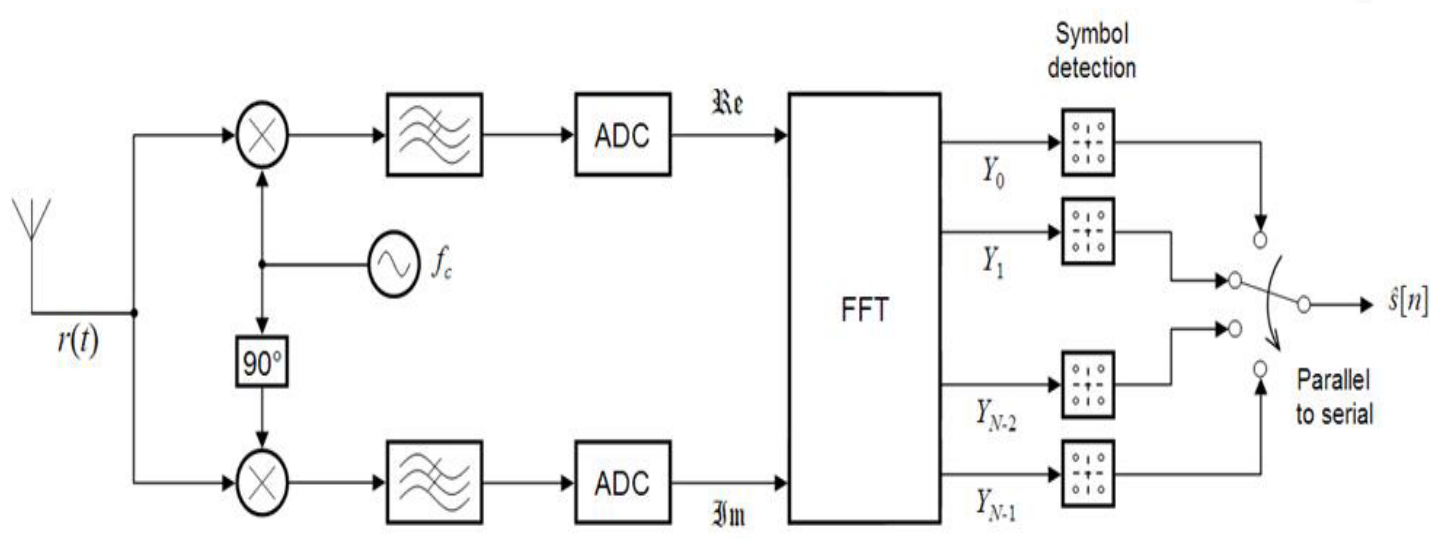

Figure 3.7: OFDM receiver. 


\subsection{OWC Optimization}

A free space optical (FSO) network consists of multiple wireless optical transceivers and receivers. This communication system is cost-effective and license free and offers high-bandwidth links. As with all OWCs, the FSO links require a line-ofsight (LOS) channel, and we will consider point-to-point connections only. They provide connection with narrow beam divergence and secure point-to-point links. Therefore, the design and optimization of this tier directly affects these factors, and optimizing them will ensure more reliable and affordable communication links [47].

\subsubsection{FSO Channel Model}

The typical FSO communication system uses intensity modulation with direct detection (IM/DD) with On-Off Keying (OOK). However, several factors affect the FSO transmission as stated above (e.g., attenuation, atmospheric turbulence, and light source tracking). The received light intensity is marginally distributed and can be estimated as follows:

$$
F_{I}(I)=\frac{1}{2 \sigma_{x} I} \frac{1}{\sqrt{2 \pi}} \exp \left\{-\frac{\left(\ln (I)-\ln \left(I_{0}\right)\right)^{2}}{8 \sigma_{x}^{2}}\right\}
$$

, where $I_{0}$ is the average received intensity, $\sigma_{x}^{2}$ is the variance of the log-amplitude uctuation, and the variance is calculated as:

$$
\sigma_{x}^{2}=0.30545\left(\frac{2 \pi}{\lambda}\right)^{7 / 6} C_{n}^{2}(\eta) z^{11 / 6}
$$

, where $\lambda$ is the wavelength, $C_{n}^{2}(\eta)$ is the parameter of the refraction structure, and its range from $10^{-13} \mathrm{~m}^{-2 / 3}$ to $10^{-17} \mathrm{~m}^{-2 / 3}$ with constant altitude $\eta$, and $z$ is the transmission distance for atmospheric channels near the ground. Moreover, the 
reliability of this link can be approximated to:

$$
\Gamma_{i j}=\operatorname{Pr}\left\{I \geq I_{t h}\right\}=1 / 2-1 / 2 \operatorname{erf}\left(\frac{\ln \left(I_{t h}\right)-\ln \left(I_{0}\right)}{2 \sigma_{x} \sqrt{2}}\right)
$$

, where $I_{t h}$ is the received signal intensity threshold. Furthermore, we define the

weight of an FSO link as: $\omega_{i j}=\Gamma_{i j}$ for $\Gamma_{i j} \geq \Gamma_{\text {th }}$ and 0 otherwise. We also assume that the links are symmetric, therefore $\Gamma_{i j}=\Gamma_{j i}$ foralli $\neq j$.

Therefore, the FSO network can be modeled as a weighted graph $G(V, E)$, while $V$ is the set of cluster heads and $E$ represents the set of FSO links. The main factors affecting FSO link weights are transmission distance and atmospheric turbulence.

\subsubsection{Optimizing the FSO Links}

Now we will bind the problem of optimizing the FSO links and the lower tier of nodes and cluster heads. The model of the FSO is a function of $V$ and $E$, with $|V|=n_{c}$ as the number of clusters and $|E|=m$ as the number of cluster heads. To measure and evaluate the reliability of the FSO links, we will use the algebraic connectivity principle.

The algebraic connectivity for a modeled graph is defined as the smallest second eigenvalue of the Laplacian matrix $\mathbf{L}$ of graph $G$. It is represented as:

$$
\lambda_{2}(L) \quad \text { and } \quad L=L(G)
$$

Therefore, and from the Laplace properties, the following characteristics can be driven:

1. The connectivity is true if and only if $\lambda_{2}(L)>0$.

2. The connectivity is false when the eigenvalues of $\mathrm{L}(\mathrm{G})$ are zero. 


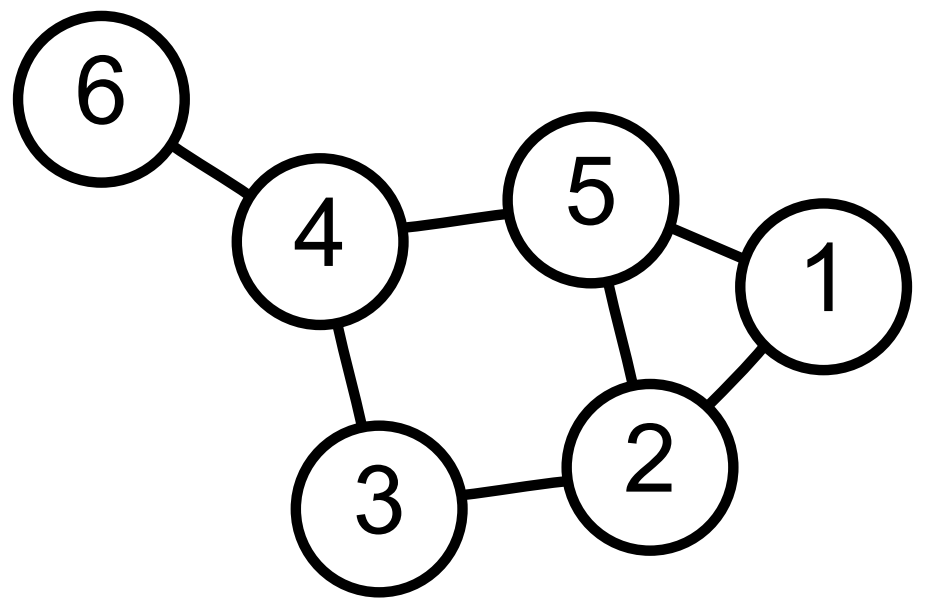

Figure 3.8: Algebraic connectivity.

3. If there are two graphs $G_{1}\left(V, E_{1}\right)$ and $G_{2}\left(V, E_{2}\right)$ with $E_{1} \subseteq E_{2}$, then $\lambda_{2}\left(L_{1}\left(G_{1}\right)\right) \leq$ $\lambda_{2}\left(L_{2}\left(G_{2}\right)\right)$.

Moreover, we can limit the algebraic connectivity by the following relationship:

$$
\frac{4}{D .|V(G)|} \leq \lambda_{2}(G) \leq \delta(G)
$$

, where $D$ is the graph diameter and $\delta(G)$ is the minimum degree of the graph. Figure 3.8 shows a simple example of the algebraic connectivity principle with 6 vertices, diameter 3 , connectivity 1 , and algebraic connectivity 0.722 [48].

The FSO optimization problem for the upper tier can be formulated as follows: Maximize:

$$
\lambda_{2}(L)
$$

Subject to:

$$
\begin{gathered}
m=1 / 2 \sum_{i=1}^{n_{c}} K_{i} \leq\left(\begin{array}{c}
n_{c} \\
2
\end{array}\right) \\
d_{i}=K_{i} \quad(\text { for all } i) \\
E \subseteq E_{\text {pot }}
\end{gathered}
$$


, where:

$K_{i}$ : is the degree of each cluster head vertex $v_{i} \in V$.

$E_{\text {opt }}$ : is the potential edges (cluster heads).

\subsubsection{FSO Greedy Edge-Appending (GEA)}

We need to optimize the edges of the FSO links in order to minimize the number of links and maximize the wavelength $\lambda$. The Greedy Edge-Appending (GEA) is an optimization algorithm that adds more edges to the current graph iteratively. This procedure of this algorithm is as follows:

1. Starts with $n_{c}$ vertices and zero edge.

2. $\lambda_{2}(L)$ is zero until the graph is connected.

3. Build a degree bounded minimum spanning tree algorithm to speed up the process.

4. Out of the $E_{p o t}$, the algorithm iteratively picks edges and connected to the graph.

5. GEA chooses the edge with the largest $\omega_{i j} .\left(v_{i}-v_{j}\right)^{2}$;

(a) If there is a tie, GEA chooses the edge with an endpoint of the minimum degree.

(b) If there is a tie again, GEA chooses the edge with the largest distance.

6. It takes $O\left(\left(n_{c}-1\right)^{2}\right)$ and builds the minimum spanning tree with $n_{c}-1$ edges.

However, the overall GEA complexity is:

$$
O\left(\left(n_{c}-1\right)^{2}+\left(m n_{c}+1\right) n_{c} m_{p o t}\right) .
$$


Figures 3.9 and 3.10 show the resulting algebraic connectivity after each edge is appended for a small and large networks.

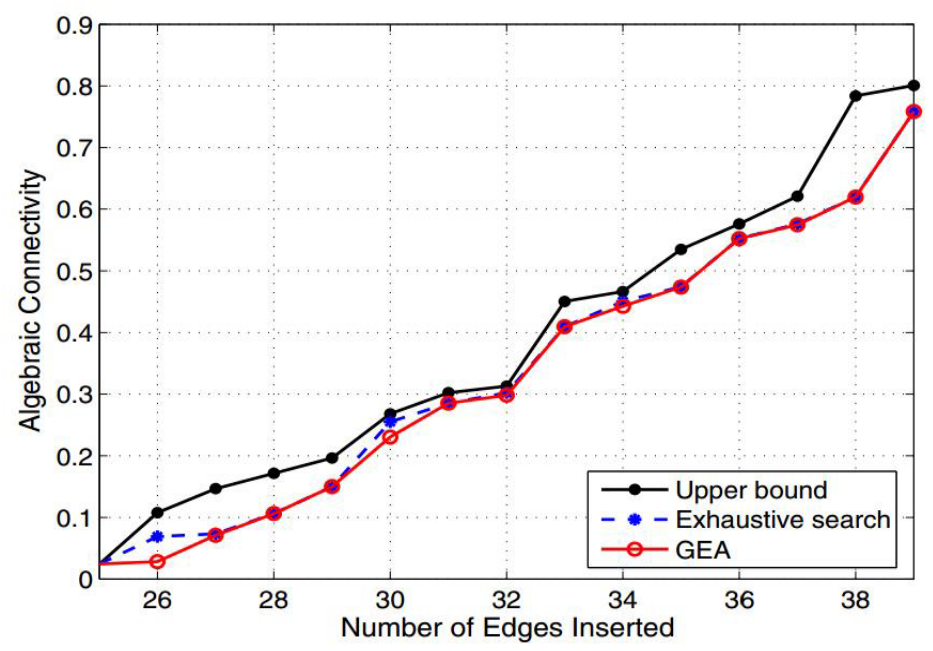

Figure 3.9: Algebraic connectivity vs. number of edges for small network.

\subsection{Summary}

In this chapter, we have discussed the optical wireless communication (OWC) networks and links. They provide high performance and reliability in the line-of-sight channels. Moreover, we discussed the use of these links and introduced the optimization techniques. The GEA algorithm has shown good simulation results compared to the lower and upper bounds. 


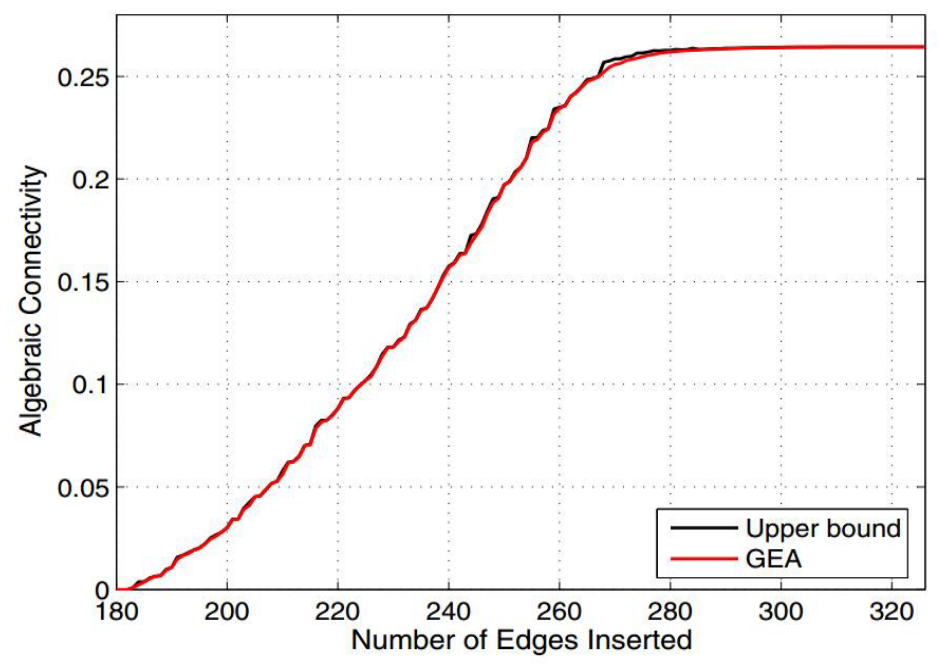

Figure 3.10: Algebraic connectivity vs. number of edges for the large network. 


\section{Chapter 4}

\section{Plane Sweeping and Clustering Algorithm (PSCA)}

\subsection{Methodology}

We will use the plane sweeping and clustering algorithm (PSCA) as in Son model [15], and try to obtain better results. The model that we will work on will be simple and then we will apply it to bigger models. The number of iterations should depend on the number of variables in the constraints. The flow chart is given in the next section. I will use Matlab to implement the technique.

\subsubsection{The Plane Sweeping and Clustering Algorithm (PSCA)}

The implementation starts with the generation of a random sample that can be replaced by a real model by defining the nodes' positions (coordinates). The model is based on the locations of the nodes in the $\mathrm{x}$ and $\mathrm{y}$ dimensions. The Matlab properties feature enabled us to build the network in a structure format. The structure format in Matlab allows arrays with named fields that can contain data of varying types and sizes. The purpose is to represent the nodes and have some features to be calculated once with each iteration. The main vector we use here is 
the identifier of the cluster number. The cluster number differentiates and clusters the nodes. The technique starts with choosing a node as a starting point. Then, the algorithm calculates the distance from each other node. If the distance is within the given limit, it adds this node to the same cluster. Then, it goes one step further by checks the new node against all the other nodes. Moreover, if the new node has a neighboring node within the specified limit, it will add it to the same cluster. If there are no more neighboring nodes, the program will jump to the next node and repeat the iteration. Since no formulas were used in the papers, we will use this iterative technique to achieve the best results in minimizing the total number of clusters. Figure 4.1 shows the technique flow chart that explains the algorithm used. And the algorithm is analyzed and implemented in table 4.1. 


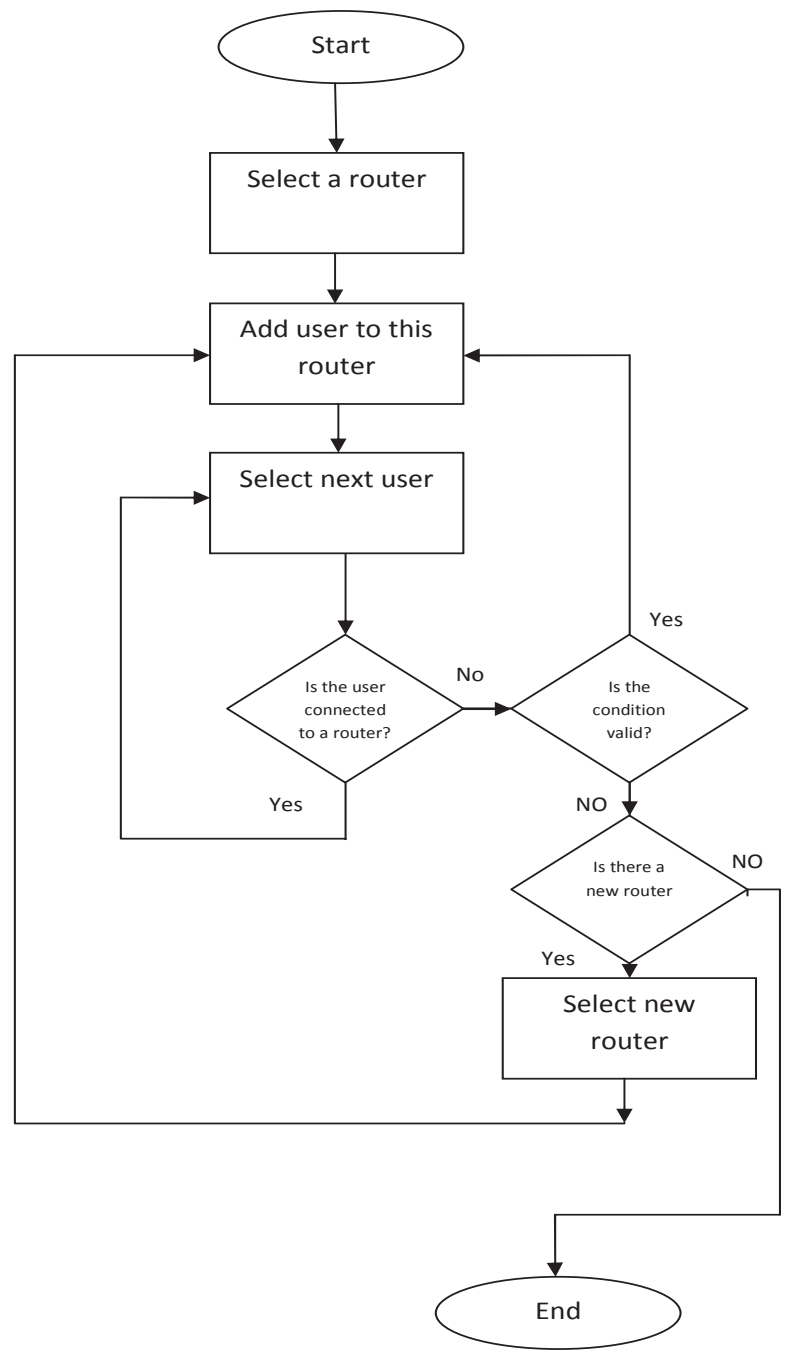

Figure 4.1: Flow chart. 
Table 4.1: The plane sweeping and clustering algorithm (PSCA).

\begin{tabular}{lc}
\hline 1 & x=n; \\
2 & Define maximum distance for communication \\
3 & Define the number of iterations \\
4 & Generate the nodes in x and y dimensions \\
5 & for (all nodes) \\
6 & Set all the nodes to cluster 0 \\
7 & Calculate the separation distance between nodes \\
8 & for (all nodes not assigned) \\
9 & Set the first node to cluster 1 \\
10 & Check this node separation with all other nodes \\
11 & while $\left(h \leq h_{\max }\right.$ ) \\
12 & Add this node to the same cluster \\
13 & while $\left(h \leq h_{\max }\right)$ \\
14 & Check for 2-hop nodes \\
15 & Add this node to the same cluster \\
16 & end while \\
17 & end while \\
18 & for (each cluster) \\
19 & Calculate the cluster gravity centre \\
20 & Plot the nodes, cluster head and cluster coverage \\
\hline
\end{tabular}




\subsubsection{Gravity Centre}

The theory of gravity centre can be used to choose central and base nodes. This theory will give the coordinates of the best location for all the nodes in a cluster. Then, by calculating the minimum distance between this virtual point and the nodes in the cluster, we can determine the base node.

$$
\begin{gathered}
\text { Distance }=\sqrt{\left(\left(x_{2}-x_{1}\right)^{2}+\left(y_{2}-y_{1}\right)^{2}\right)} \\
X_{c}=\sum_{i} \frac{x_{i} \cdot w_{i}}{x_{i}} \\
Y_{c}=\sum_{i} \frac{y_{i} \cdot w_{i}}{y_{i}}
\end{gathered}
$$

The centre of gravity of each cluster will determine the best position to mount the base station. it will reduce the amount of power needed and will ensure the most coverage for the network.

\subsection{Simulations}

The algorithm shows good results in analyzing the network and taking into account the constraints, the number of hops, and the maximum distance. The algorithm was applied to a number of random network constellations and it shows some improved results. In the simulation we will use different network setups. The nodes will be generated with the specified number and distributed randomly. The are used can be specified in the algorithm and as well as the ranging of the nodes. 


\subsubsection{Simulation A}

In the first simulation (figures 4.2, 4.3), a network of 20 nodes was introduced and the algorithm showed six clusters. The constraints were:

1. Number of nodes $=20$

2. Maximum number of hops $=2$

3. Maximum distance $=3$

4. Network size $=10 \times 10$

The first figure shows the entire network that to be distributed. Then, each cluster is represented in a separate figure. The blue circle represents a node (router). The red square represents the centre of gravity and the circle shows the adaptive range. See figure 4.3 .

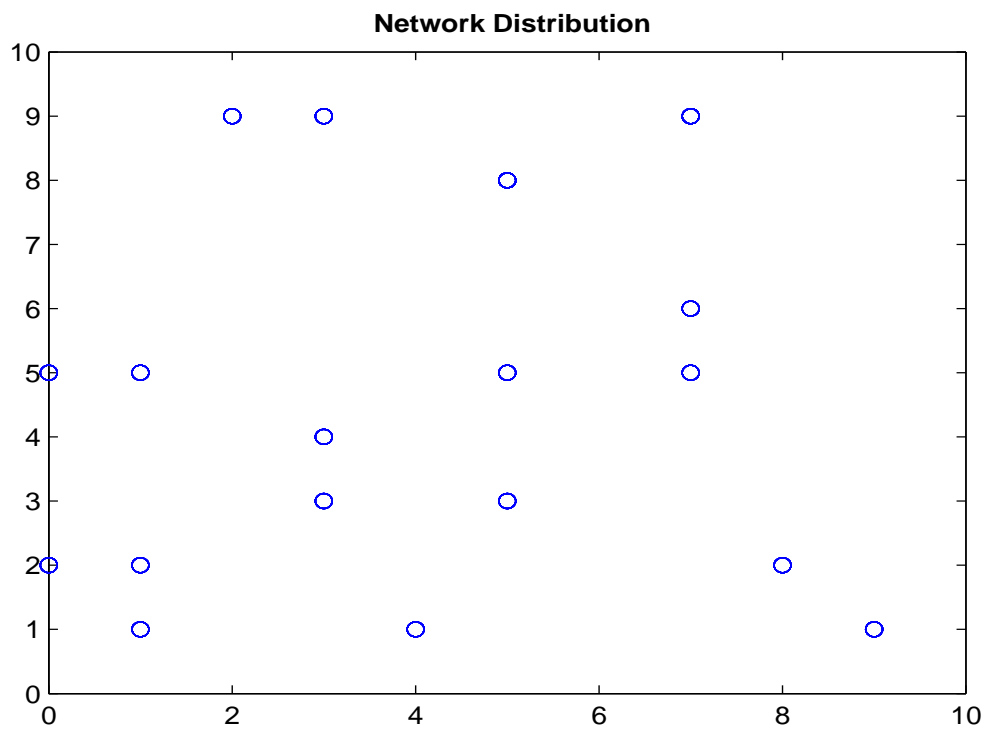

Figure 4.2: Simulation A: network distribution. 

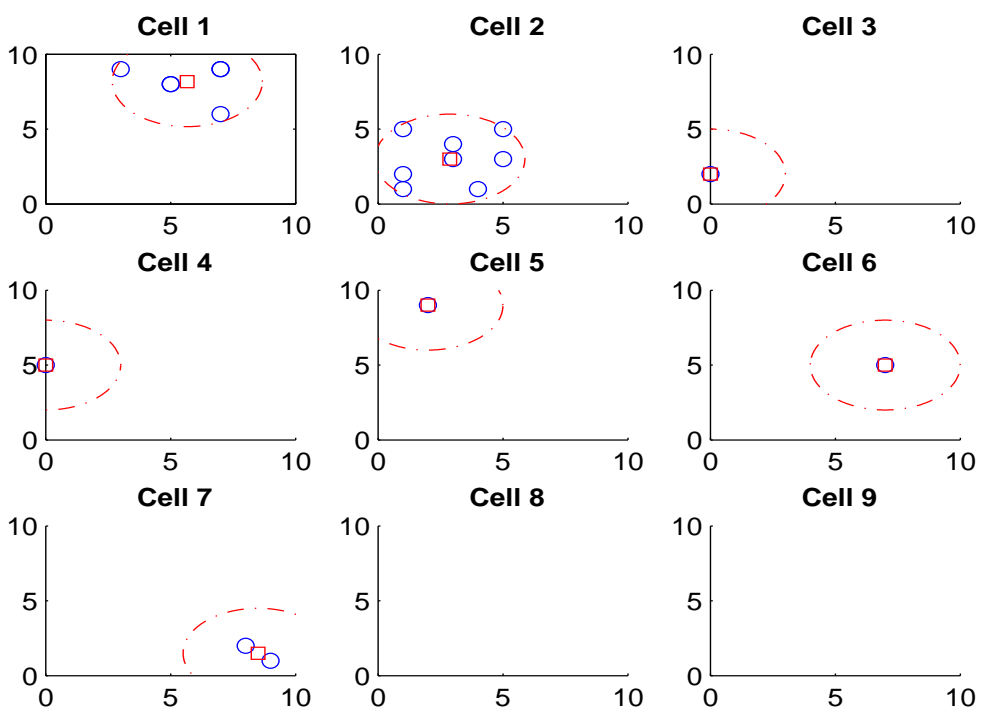

Figure 4.3: Simulation A: clusters.

\subsubsection{Simulation B}

In this simulation (figures 4.4, 4.5), a new network of 30 nodes was introduced and the algorithm showed eight clusters. The constraints were:

1. Number of nodes $=30$

2. Maximum number of hops $=2$

3. Maximum distance $=3$

4. Network size $=10 \times 10$ 


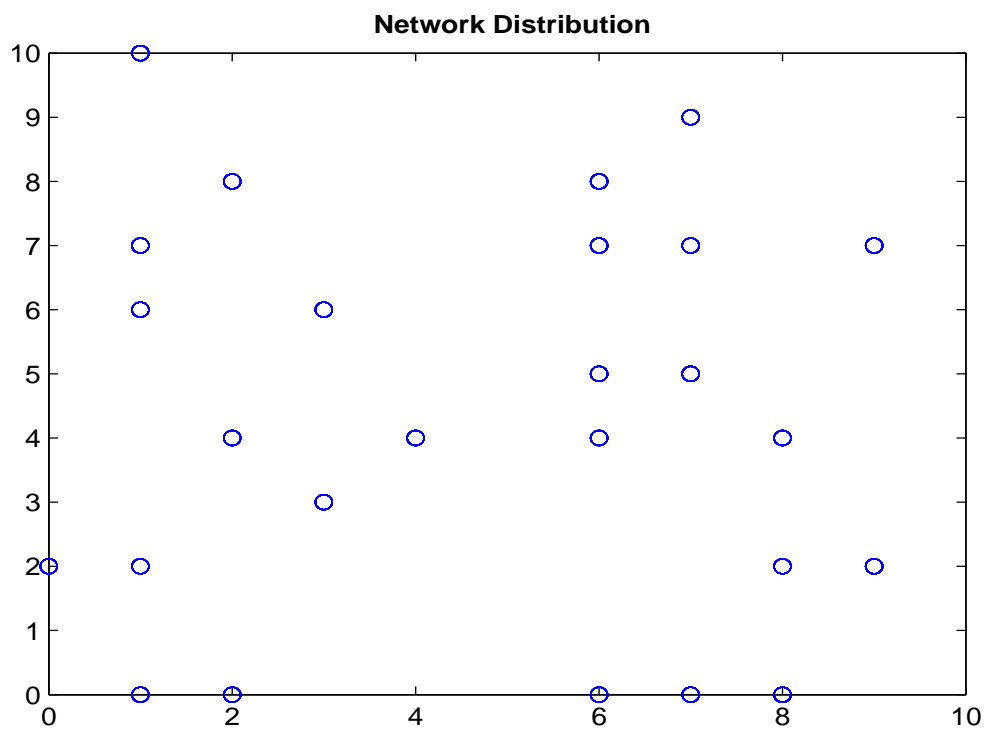

Figure 4.4: Simulation B: network distribution.
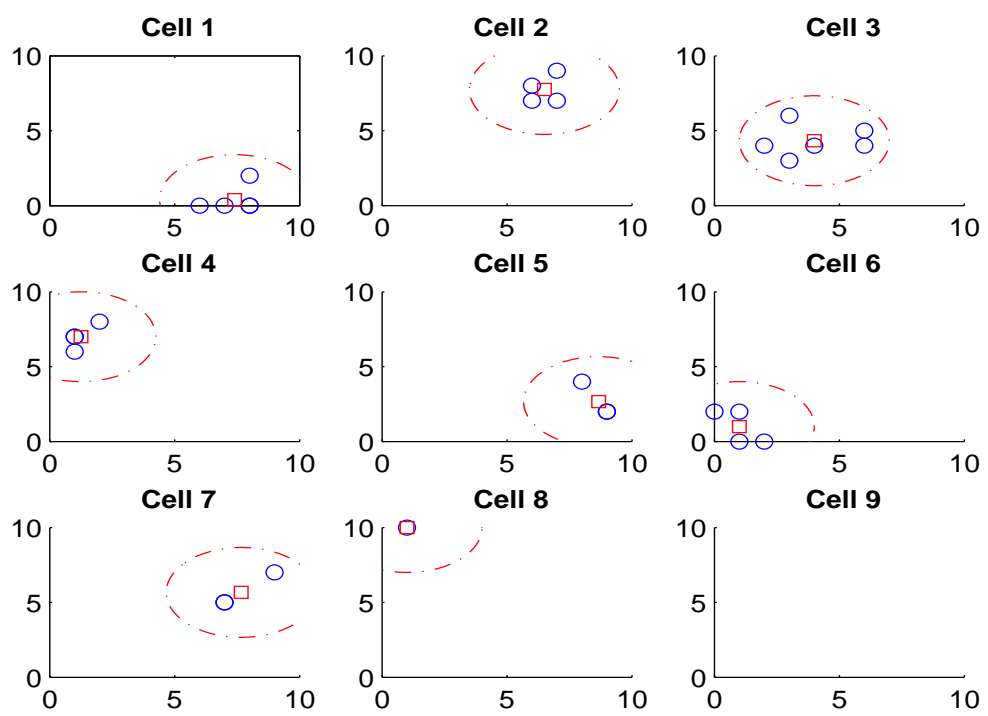

Figure 4.5: Simulation B: clusters. 


\subsubsection{Simulation C}

In this simulation we will increase the implementation area and the number of users as well with changing the maximum distance for each tower (figures 4.6, 4.7), a new network of 50 nodes was introduced and it showed nine clusters. The constraints were:

1. Number of nodes $=50$

2. Maximum number of hops $=2$

3. Maximum distance $=20$

4. Network size $=100 \times 100$

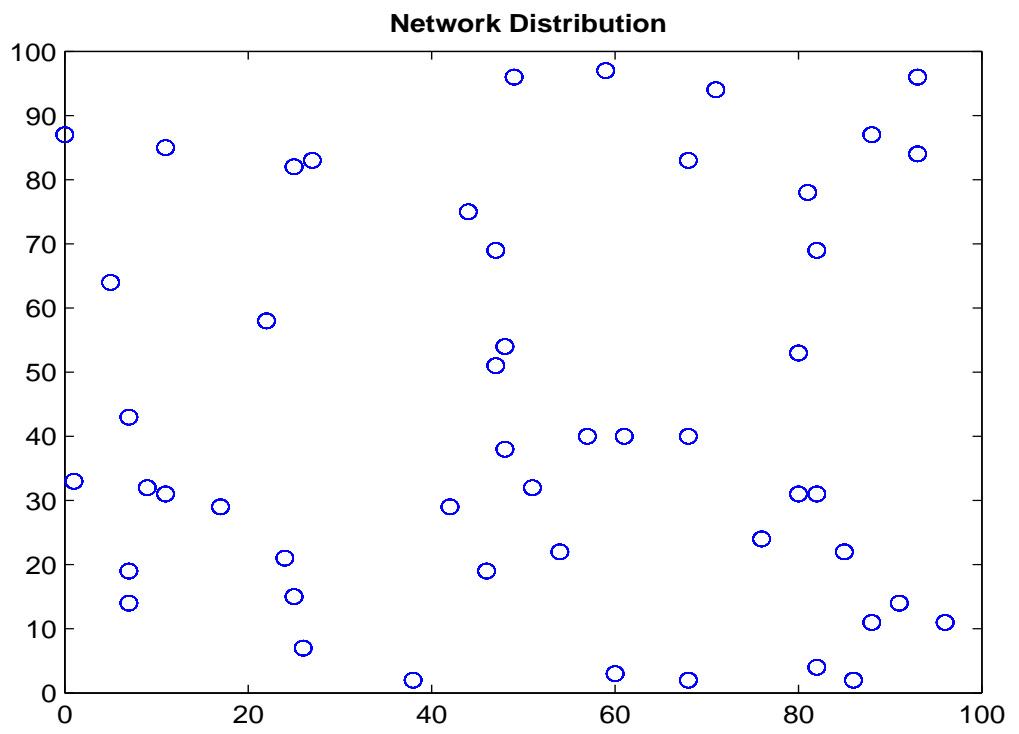

Figure 4.6: Simulation C: network distribution. 

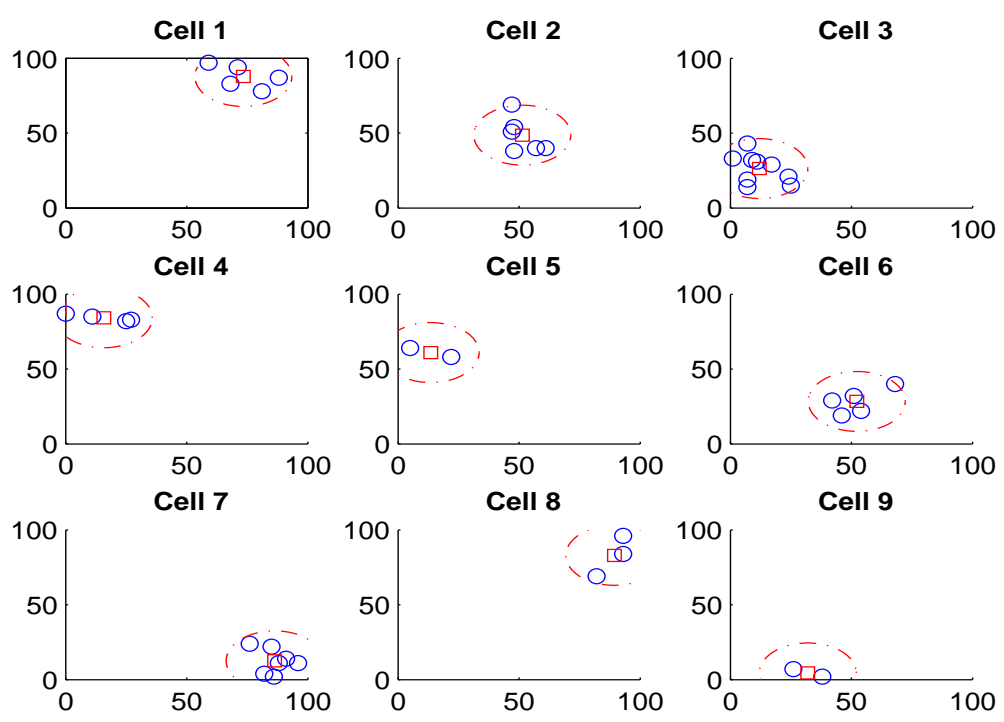

Figure 4.7: Simulation C: clusters.

\subsection{Results and Discussion}

Now we will apply this simulation for multiple iterations to compare the results with the proposed paper. We used the following parameters:

1. Number of iterations per cluster diameter $=50$

2. Number of nodes $=20$

3. Maximum number of hops $=2$

4. Maximum cluster diameter ranging [5 - 70]

Table 4.2 shows the comparison of the trend:

Graph 4.8 shows the relationship between the cluster diameter and the number of clusters. These readings were based on the mean number of 50 iterations of a random network distribution of a 20-node network. 
Table 4.2: Cluster diameter and number of clusters.

\begin{tabular}{|l|l|l|}
\hline No. & Cluster diameter & Mean number of clusters \\
\hline 1 & 5 & 18.88 \\
2 & 10 & 15.34 \\
3 & 20 & 9.88 \\
4 & 30 & 6.46 \\
5 & 40 & 4.46 \\
6 & 50 & 3.44 \\
7 & 60 & 2.7 \\
8 & 70 & 2.34 \\
\hline
\end{tabular}

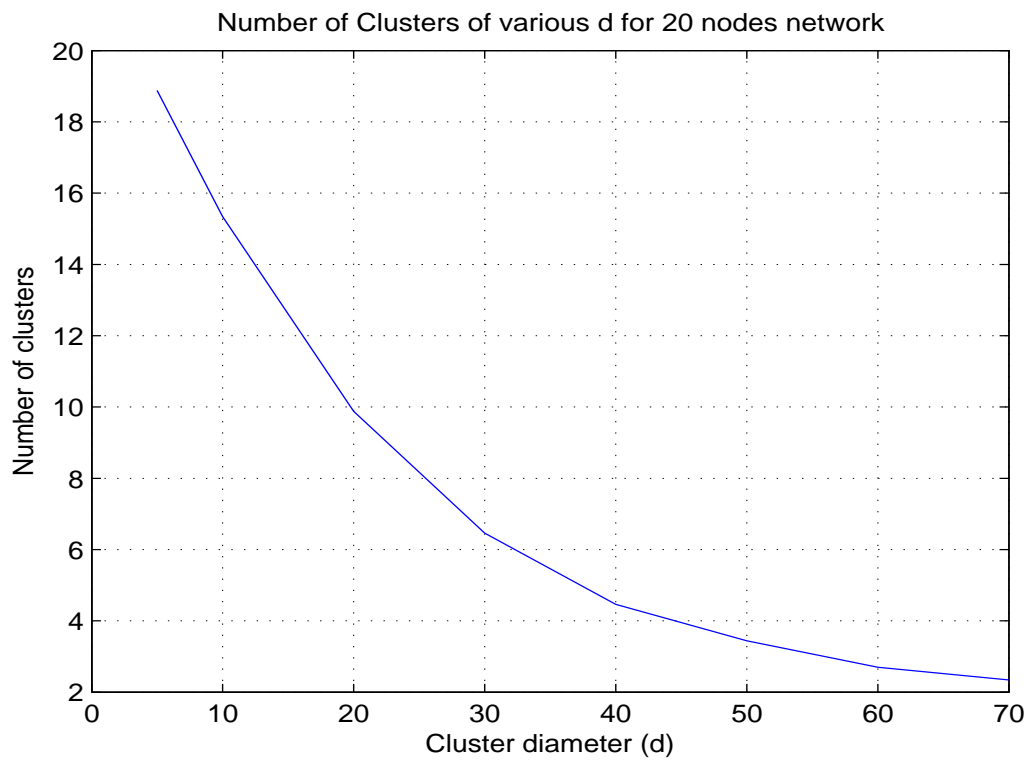

Figure 4.8: Number of clusters vs cluster diameter for 20 nodes network.

Furthermore, we added a new feature to the software to enhance the performance of the implementation. This improvement will implement the clustering on different cluster sizes (coverage diameter). The algorithm will generate a different number of combinations of cluster sizes. The implication of the changes in the diameter of the cluster can be compared easily. Graph 4.9 shows the implementation using these 
criteria from the referred paper [15] to compare the results:

1. Number of nodes $=50$

2. Maximum number of hops $=2$

3. Network size $=1000 \times 1000$

4. Maximum cluster diameter ranging [100 - 1000]

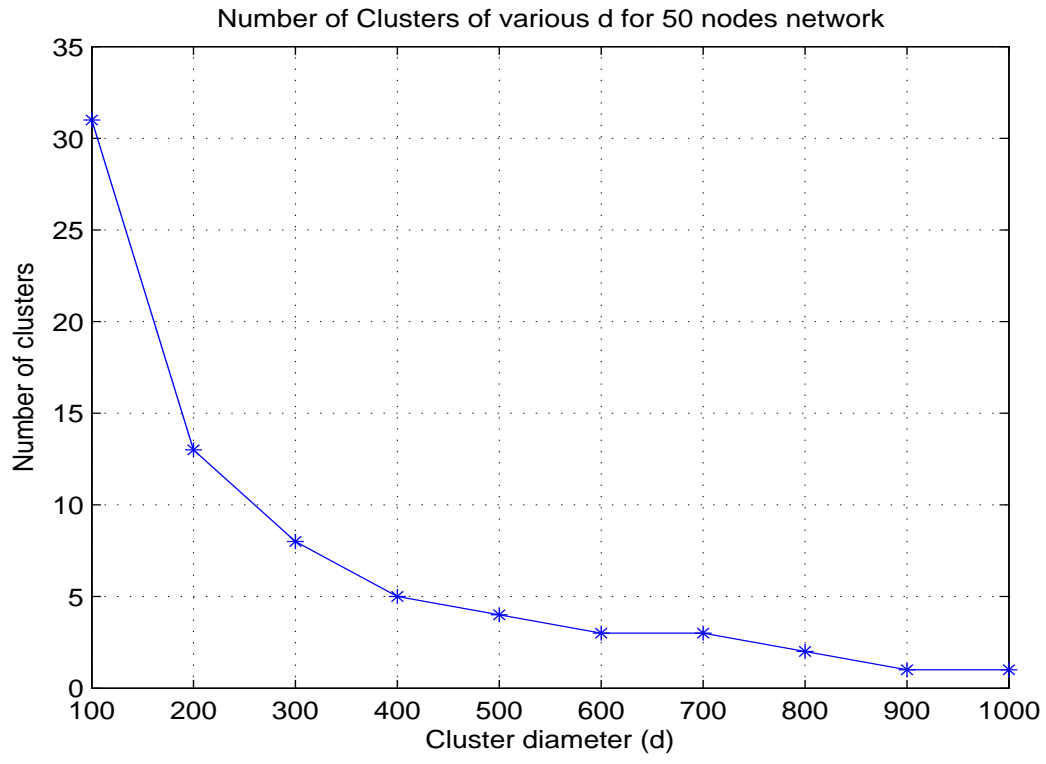

Figure 4.9: Number of clusters for 50 nodes.

The last graph 4.10 is for the 175 nodes network as compared to the same paper. 


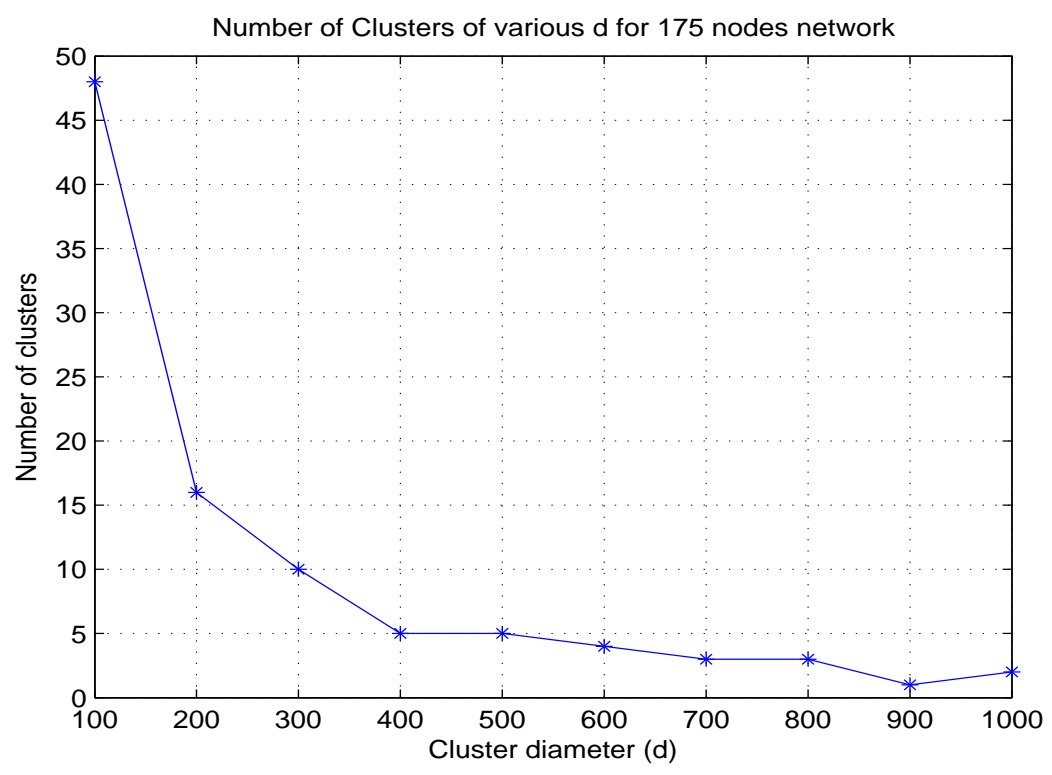

Figure 4.10: Number of clusters for 175 nodes.

The different simulations and graphs show that the algorithm is accurately clustering the network distribution in different clusters. We have obtained from the implementation both some very congested clusters and a mono-cluster or an empty one. This can cause an unfairness problem. The congested clusters will have more traffic and therefore more connection blockage and more dropped connections.

\subsection{Summary}

In this chapter, we have derived the algorithm and how it can be implemented on a wireless network distribution. Moreover, we have shown the results from the Matlab software on a different network schemes. We then compared our results with the proposed paper by implementing the same conditions on the wireless network distribution. 


\section{Chapter 5}

\section{Conclusion and Future Work}

\subsection{Conclusion}

The problem has been investigated for long time but an optimal solution is expected.

This issue is very important and the implementation in the real life is highly on demand . Therefore, the use of different techniques should give more optimization for this problem. The two-tier mesh network consists of upper and lower tiers. We discussed the upper tier with the optical wireless communication (OWC) links and networks. The optimization discussed was about the greedy edge-appending (GEA) to reduce the number of links and maximize the wavelength. MOreover, for the lower tier, we studied the plane sweeping and clustering algorithm (PSCA) to optimize the cluster size in regard to coverage and connectivity.

The results achieved can be implemented for real life applications. The gravity centre theory was used to achieve a better central point of communication in each cluster. Such applications are wireless network routers and cellular phones. Moreover, this algorithm can be developed to cover mobile networks such as vehicle wireless and wireless sensor networks.

This simulation has been implemented for the lower tier using the optimization 
algorithm of plane sweeping and clustering. The algorithm showed capability in covering all the nodes in the area and they are divided into clusters. The larger distance we cover by each cluster head, the more nodes will be covered. However, the more distance a cluster head covers, the more power it will need to reach that distance.

\subsection{Future Work}

This topic is very rich and can be extended in a variety of ways:

- Dynamic module: As an extension to this project, a dynamic module is suggested to improve optimization.The dynamic module will consider different coverage distances for each cluster head and optimize the distance based on the node's range. The module should be run several times with clusters of different sizes so that the optimal solution can be chosen.

- Power calculations: Another future work for this project can be done by adding the power calculations of the cluster heads and optimizing the power consumption based on the range and coverage of the cluster.

- Congestion: The congestion of the clusters can cause several problems and therefore may degrade the network's reliability and performance. The less congestion in the cluster, the better the reliability and availability of the service. Future research in this direction can be improved for this algorithm by taking into account the number of nodes which are covered by each cluster head. The module should check the number of nodes in each cluster and limit it to the optimal number of nodes to ensure a fair distribution and to manage the congestion. 


\section{Bibliography}

[1] I. F. Akyildiz, X. Wang, and W. Wang, "Wireless mesh networks: a survey," Computer Networks, vol. 47, no. 4, pp. 445 - 487, 2005.

[2] I. I. Kim, B. McArthur, and E. J. Korevaar, "Comparison of laser beam propagation at $785 \mathrm{~nm}$ and $1550 \mathrm{~nm}$ in fog and haze for optical wireless communications," Proc. SPIE 4214, Optical Wireless Communications III, pp. 26-37, 2001.

[3] Massachusetts Institute of Technology, "Free space optical network." http :

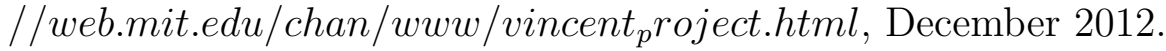

[4] ITU, "Statistical highlights." http://www.itu.int/ITU-D/ict/statistics/, July 2012 .

[5] A. G. J. Andrews and R. Muhamed, Fundamentals of WiMAX; understanding broadband wireless networking, vol. 31. Prentice Hall, 2007.

[6] I. Akyildiz, W. Su, Y. Sankarasubramaniam, and E. Cayirci, "Wireless sensor networks: a survey," Computer Networks, vol. 38, no. 4, pp. 393 - 422, 2002.

[7] S. Basagni, "Distributed clustering for ad hoc networks," in Fourth International Symposium on Parallel Architectures, Algorithms, and Networks, 1999. (I-SPAN '99) Proceedings., pp. 310 -315, 1999.

[8] M. Qin and R. Zimmermann, "An energy-efficient voting-based clustering algorithm for sensor networks," in SNPD/SAWN 2005., pp. 444 - 451, may 2005. 
[9] M. Soysa, "Performance of dual hop relay systems with imperfect csi," MASc, University of Alberta, Fall 2011.

[10] IEEE Computer Society Std., IEEE Standard for Information technology Telecommunications and informa- tion exchange between systems Local and metropolitan area networks Spe- cific requirements Part 11: Wireless LAN Medium Access Control (MAC) and Physical Layer (PHY) Specifications, Nov. 2007.

[11] IEEE Computer Society Std., IEEE Standard for Information technology Telecommunications and information exchange between systems Local and metropolitan area networks Specific requirements Part 15.1: Wireless Medium Access Control (MAC) and Physical Layer (PHY) Specifications forWireless Personal Area Networks (WPANs), Jun. 2005.

[12] IEEE Computer Society and the IEEE Microwave Theory and Techniques Society, IEEE Recommended Practice for Local and metropolitan area networks Coexistence of Fixed Broadband Wireless Access Systems, 2004.

[13] L. Miller, "Probability of a two-hop connection in a random mobile network," in Proc. 35th Conf. on Information Sciences and Systems (CISS 2001), 2001.

[14] V. Sreng, H. Yanikomeroglu, and D. Falconer, "Coverage enhancement through two-hop relaying in cellular radio systems," in Wireless Communications and Networking Conference WCNC, vol. 2, pp. 881 - 885, mar 2002.

[15] I. Son and S. Mao, "Design and optimization of a tiered wireless access network," in Proceedings IEEE INFOCOM, pp. 1 -9, march 2010.

[16] V. Veeravalli and A. Sendonaris, "The coverage-capacity tradeoff in cellular cdma systems," IEEE Transactions on Vehicular Technology, vol. 48, pp. 1443 -1450 , sep 1999. 
[17] R. Draves, J. Padhye, and B. Zill, "Routing in multi-radio, multi-hop wireless mesh networks," in Proceedings of the 10th annual international conference on Mobile computing and networking, pp. 114-128, ACM, 2004.

[18] J. Jun and M. Sichitiu, "The nominal capacity of wireless mesh networks," IEEE Wireless Communications, vol. 10, no. 5, pp. 8-14, 2003.

[19] J. Shi, O. Gurewitz, V. Mancuso, J. Camp, and E. Knightly, "Measurement and modeling of the origins of starvation in congestion controlled mesh networks," in INFOCOM 2008. The 27th Conference on Computer Communications, pp. 1633-1641, IEEE, 2008.

[20] M. Alicherry, R. Bhatia, and L. E. Li, "Joint channel assignment and routing for throughput optimization in multi-radio wireless mesh networks," in Proceedings of the 11th annual international conference on Mobile computing and networking, MobiCom '05, (New York, NY, USA), pp. 58-72, ACM, 2005.

[21] B. Liu, Z. Liu, and D. Towsley, "On the capacity of hybrid wireless networks," in INFOCOM 2003. Twenty-Second Annual Joint Conference of the IEEE Computer and Communications, vol. 2, pp. 1543-1552, 2003.

[22] G. Bolch, S. Greiner, H. de Meer, and K. Trivedi, Queueing networks and Markov chains: modeling and performance evaluation with computer science applications. Wiley-Interscience, 2006.

[23] I. F. Akyildiz, D. M. Gutierrez-Estevez, and E. C. Reyes, "The evolution to 4G cellular systems: LTE-advanced," Physical Communication, vol. 3, no. 4, pp. $217-244,2010$.

[24] N. Tripathi, J. Reed, and H. VanLandinoham, "Handoff in cellular systems," Personal Communications, IEEE, vol. 5, no. 6, pp. 26-37, 1998.

[25] S. Tekinay and B. Jabbari, "Handover and channel assignment in mobile cellular networks," IEEE Communications Magazine, vol. 29, no. 11, pp. 42-46, 1991. 
[26] A. Acampora and M. Naghshineh, "Control and quality-of-service provisioning in high-speed microcellular networks," IEEE Personal Communications, vol. 1, no. 2, p. 36, 1994.

[27] I. Akyildiz, J. Xie, and S. Mohanty, "A survey of mobility management in next-generation all-ip-based wireless systems," IEEE Wireless Communications, vol. 11, no. 4, pp. 16-28, 2004.

[28] A. of the CAR 2 CAR Communication Consortium, "http://www.car-tocar.org/ @ONLINE," 2012.

[29] A. Marshall, "V2V: GM technology can prevent accidents," GM Europe, 2006.

[30] IEEE 5.9 GHz Dedicated Short Range Communications (DSRC), "http : //grouper.ieee.org/groups/scc32/toplvl3.html/@ONLINE,” Dec. 2012.

[31] I. Chisalita, Communication and Networking Techniques for Traffic Safety Systems. PhD thesis, Linkping University, IISLAB, The Institute of Technology, 2006.

[32] W. Alasmary and O. Basir, "Achieving efficiency and fairness in 802.11-based vehicle-to-infrastructure communications," in Vehicular Technology Conference (VTC Spring), 2011 IEEE 73rd, pp. 1 -6, may 2011.

[33] A. Abbasi and M. Younis, "A survey on clustering algorithms for wireless sensor networks," Computer communications, vol. 30, no. 14, pp. 2826-2841, 2007.

[34] C.-F. Huang and Y.-C. Tseng, "The coverage problem in a wireless sensor network," Mobile Network Application, vol. 10, pp. 519-528, Aug. 2005.

[35] K. Sohrabi, J. Gao, V. Ailawadhi, and G. Pottie, "Protocols for selforganization of a wireless sensor network," IEEE Personal Communications, vol. 7 , no. 5, pp. 16-27, 2000. 
[36] Y. Cheng and D. P. Agrawal, "An improved key distribution mechanism for large-scale hierarchical wireless sensor networks," Ad Hoc Networks, vol. 5, no. 1, pp. $35-48,2007$.

[37] S. G. R. Dutta and M. K. Das, "Efficient statistical clustering techniques for optimizing cluster size in wireless sensor network," Procedia Engineering, vol. 38, pp. 1501 - 1507, 2012. International Conference on Modeling, Optimization and Computing.

[38] K. Gerd, Optical fiber communications. Mc Graw Hill, 4 ed., 2011.

[39] K. Wang, A. Nirmalathas, C. Lim, and E. Skafidas, "High-speed optical wireless communication system for indoor applications," IEEE Photonics Technology Letters, vol. 23, no. 8, pp. $519-521,2011$.

[40] A. Mahdy and J. Deogun, "Wireless optical communications: a survey," in IEEE Wireless Communications and Networking Conference, vol. 4, pp. 2399 - 2404 Vol.4, march 2004.

[41] L. Andrews and R. Phillips, Laser beam propagation through random media, vol. 152. Society of Photo Optical, 2005.

[42] X. Zhu and J. Kahn, "Free-space optical communication through atmospheric turbulence channels," IEEE Transactions on Communications, vol. 50, pp. 1293 - 1300, aug 2002.

[43] D. Kedar and S. Arnon, "Urban optical wireless communication networks: the main challenges and possible solutions," IEEE Communications Magazine, vol. 42, pp. S2 - S7, may 2004.

[44] D. Kedar and S. Arnon, "Optical wireless communication through fog in the presence of pointing errors," Appl. Opt., vol. 42, pp. 4946-4954, Aug 2003. 
[45] J. Andrews, A. Ghosh, and R. Muhamed, Fundamentals of WiMAX: understanding broadband wireless networking. Prentice Hall PTR, 2007.

[46] Y. Cho, J. Kim, W. Yang, and C. Kang, MIMO-OFDM wireless communications with MATLAB. Wiley, 2010.

[47] H. Sandalidis, T. Tsiftsis, and G. Karagiannidis, "Optical wireless communications with heterodyne detection over turbulence channels with pointing errors," Journal of Lightwave Technology, vol. 27, pp. 4440 -4445, oct.15, 2009.

[48] Wikipedia, "Algebraic connectivity." //www.en.wikipedia.org/wiki/Algebraic onnectivity/, December 2012. 